\title{
COVID19: A Natural Language Processing and Ontology Oriented Temporal Case-Based Framework for Early Detection and Diagnosis of Novel Coronavirus
}

\author{
Olaide N. Oyelade ${ }^{1,2}$, Absalom E. Ezugwu ${ }^{2}$ \\ ${ }^{1}$ Department of Computer Science, Ahmadu Bello University Zaria, Nigeria \\ ${ }^{2}$ School of Computer Science, University of KwaZulu-Natal, King Edward Avenue, Pietermaritzburg Campus, \\ Pietermaritzburg, 3201, KwaZulu-Natal, South Africa \\ Corresponding authors emails: \{olaide_oyelade@yahoo.com,ezugwua@ukzn.ac.za\}
}

\begin{abstract}
:
Coronavirus, also known as COVID-19, has been declared a pandemic by the World Health Organization (WHO). At the time of conducting this study, it had recorded over 1.6million cases while more than 105,000 have died due to it, with these figures rising on a daily basis across the globe. The burden of this highly contagious respiratory disease is that it presents itself in both symptomatic and asymptomatic patterns in those already infected, thereby leading to an exponential rise in the number of contractions of the disease and fatalities. It is therefore crucial to expedite the process of early detection and diagnosis of the disease across the world. The case-based reasoning (CBR) model is an effective paradigm that allows for the utilization of cases' specific knowledge previously experienced, concrete problem situations or specific patient cases for solving new cases. This study therefore aims to leverage the very rich database of cases of COVID-19 to interpret and solve new cases even at their early stage to the advanced stage. The approach adopted in this study employs a natural language processing (NLP) technique to parse records of cases and thereafter formalize each case which is represented as a mini-ontology file. The formalized case is therefore parsed into a CBR model to allow for classification of the case into positive or negative to COVID-19. Meanwhile, feature extraction for each case is done by classifying tokens extracted by the NLP approach into special, temporal and thematic classes before encoding them using an ontology modeling method. The CBR model therefore leverages on the formalized features to compute the similarity of the new case with extracted similar cases from the archive of the CBR model. The proposed framework was populated with 68 cases obtained from the Italian Society of Medical and Interventional Radiology (SIRM) repository. Results obtained revealed that the proposed approach leverages on locations (spatial) and time (temporal) of contagion to successfully detect cases even in their early stages of two days onward before the incubation period of fourteen days. The proposed framework achieved an accuracy of $97.10 \%$, sensitivity of 0.98 and specificity of .066 . The study found that the proposed model can assist physicians to easily diagnose and isolate cases, thereby minimizing the rate of contagion and reducing false diagnosis as observed in some parts of the globe.
\end{abstract}

Keywords: Coronavirus, case-based reasoning, ontology, natural language processing.

\subsection{Introduction}

The novel coronavirus disease, also referred to as COVID-19, was first identified in China in December 2019. The virus has so far affected 213 countries and territories around the world and 2 international conveyances and is now considered a major global health concern due to its pathogenicity and widespread distribution around the world. The Covid-19 virus is a highly contagious respiratory disease that has raced around the globe since it first emerged in China in late December 2019. According to the World Health Organisation's official reports on COVID-19 [13], by April 21, 2020, it had affected more than 2,482,598 million people and caused more than 170,484 deaths, with a total of 652,543 recovered. Considering the exponential growth in the confirmed and death cases of COVID-19, this has expedited efforts by the scientific and research community in proposing and developing several novel epidemiological model approaches to mitigate the spread of the COVID-19 outbreak. 
A number of mathematical and statistical models have been developed recently to critically analyze the transmission pattern of the ongoing COVID-19 and other related disease outbreaks [14, 15, 16, 17, 18, 19]. It is equally important to recognize all the different epidemiological contributions towards estimating the transmission dynamics of the virus, but most of the existing proposed models are parameter dependent and they rely mainly on multiple assumptions [20] for them to be effective. Moreover, because during an outbreak of any epidemic, it is often not easy and reliable to estimate parameters using real data sets, which are not readily available for experimental testing of such proposed models [21, 22]. Furthermore, in most of the reported model parameter settings, one can discover that rather than using the actual parameter values that seem close enough to the real-world values derived from the statistical properties of the actual data sets, the authors of those models opted to use hypothesized parameter values. However, the use of hypothesized parameters in this case is highly limited because it does not fit the data very well [20]. Therefore, considering the aforementioned challenges associated with the current existing mathematical and statistical epidemiological models, it would be very difficult to attribute any high predictive accuracy level for using these models to correctly estimate and forecast the exponential growth of COVID-19 outbreaks. As it stands for now, despite all these measures and attractive modeling proposals, the virus has maintained its capacity to spread exponentially from country to country and continent to continent stretching the functionality and capability of even the most robust healthcare systems of so many countries.

Although many related artificial intelligence (AI) based proposed studies in the literature appear to be well-designed for the tasks of handling the current coronavirus pandemic in terms of estimating confirmed cases and forecasting the speed of COVID-19 spread, these models may deteriorate in performance and accuracy due to their heavy reliance on many inaccurate decision variables and imprecise parameter estimations. Thus, it is assumed that the aforementioned limitations can lead to conflicting forecasting outcomes, which may invariably lead to unsatisfactory and imprecise results. This would obviously have a negative impact on public health planning and policy making. To overcome the limitations of the aforementioned existing epidemiological and AI-based model approaches, the current paper presents a promising alternative diagnostic and forecasting framework with the aim to achieve more accurate results and avoid the previous limitations by combining the strengths of ontology-based natural language processing with case-based reasoning for early detection and diagnosis of the novel coronavirus pandemic. The rich database of cases of confirmed COVID-19 supports the adoption of case-based reasoning (CBR) paradigm as an authentic reasoning structure for improving diagnosis.

Case based reasoning (CBR) is an artificial intelligence paradigm that has proven to be effective in medical systems, and also exploits the similarity of cases in its knowledge base in providing a solution to a new case or problem. Case(s) retrieval that is closely related with the new case is usually computed using different similarity computational models like Euclidean distance which have been adopted by different researches. However, CBR systems all have the challenge of features extraction and formalization. Furthermore, the choice of selecting the best distance measure model for computing similarity of cases is a problem demanding optimal solution considering the sensitivity of medical cases. Case-based reasoning means using old experiences to understand and solve new problems. In case-based reasoning, a reasoner remembers a previous situation similar to the current one and uses that to solve the new problem [59]. Case-based reasoning (CBR) and expert systems have a long tradition in artificial intelligence. CBR has been formulated since the late 1970s. CBR is an approach for problem solving and learning of humans and computers [60]. Case-based reasoning is useful in problem solving and automation of learning by an agent. Because empirical evidence has shown that reasoning with CBR is more powerful, this has made reasoning by re-using past cases a powerful and frequently applied way to solve problems for humans. A very important feature of case-based reasoning is its coupling to learning, and its strong association with machine learning [61]. Ben-Bassat, et al. [62] enumerated some features of CBR, and these include: cases that present similar symptoms and findings results from same faults/disease, and "Nearest Neighbor" algorithm is used to identify unknown diagnosis from the known. CBR is beneficial when compared to RBR. CBR avoids the knowledge based acquisition bottleneck of RBR, it compiles past solutions, mimics the diagnostic experience of human experts, avoids past mistakes, interprets rules, supplements weak domain models, facilitates explanation, supports knowledge acquisition and learning, and exploits the database of solved problems so as to learn.

In this paper, we introduce the concept of combining machine learning and artificial intelligence techniques to the most important challenges in responding to the novel coronavirus or COVID-19 pandemic. Consequently, the main goal of this study is to apply the concept of natural language processing (NLP) based CBR technique to the problem of classifying cases of COVID-19 as either positive or negative even when the disease is still in its early stage of manifestation in the presented case. An NLP model for feature extraction of presented case was designed and 
implemented. The originality of the current study lies in the robustness and efficiency of the sentence-level extraction of feature-value pair for all a-priori declared features. Furthermore, the case retrieval similarity metric applied to the proposed NPL-based CBR framework contributes to the interesting performance of the proposed system. Specifically, the technical contributions of this study are as follows:

i. Adapt standard NLP techniques and libraries for carrying out data/input pre-processing. This allows for the cleaning and reformatting of natural language-based patient records using NLP approach.

ii. The pre-processed data was then applied to techniques for extraction of features of COVID-19 disease. This extracted feature provides the CBR framework with tokens as input to support case-based operations.

iii. To provide support for computational data representational approach, we formalized the extracted features using ontologies.

iv. We developed a CBR framework which allows for detecting/classifying suspected cases COVID-19 as either positive or negative.

The remainder of the paper is organized into six sections, namely: the related works, proposed approach, experimentation, results, discussion, and conclusion. The related works section presents a comprehensive review of related studies and bibliometric analysis study of research activities on COVID-19. In Section 3, a detail of the approach proposed for the CBR framework is presented, while Section 4 discusses the experimentation and system configuration for the experimentation. In Section 5, we present a comparison of the performance of the proposed approach with some related studies, and then conclude the study in Section 6.

\subsection{Related Works}

This section consists of two parts; the first part covers the detailed review of all the current related work that has so far been implemented to handle the ongoing novel coronavirus. The second part deals with the aspect of bibliometric analysis of the existing literature on COVID-19.

\subsection{Related Work}

In recent times, artificial intelligence (AI) has been considered as a potentially powerful tool in the fight against many evolving pandemics such Ebola hemorrhagic fever (2014-2016), Swine flu (2002-2003), SARS (2002-2003), Middle East respiratory syndrome coronavirus (MERS-CoV) (2012-present), and novel coronavirus (COVID-19) (2019-ongoing). Regarding the ongoing 2019-2020 novel coronavirus pandemic, dozens of research efforts have emerged and most of the published papers focused on the important of harnessing artificial intelligence technologies to curb the global COVID-19 Pandemic. This section provides a selective review of recent articles that have discussed the many significant contributions of the application of AI technologies in the fight against COVID-19, as well as the current constraints on these contributions. Specifically, in [1], six areas where artificial intelligence technologies have emerged as key solutions to combatting coronavirus were identified. These areas include: i) early warnings and alerts, ii) tracking and prediction, iii) data dashboards, iv) diagnosis and prognosis, v) treatments and cures, and vi) social control. Therefore, most of the subsequent discussions presented in this section are focused on investigating to what extent AI has been partly or fully utilized in combatting the spread of the aforementioned pandemic. The selected review discussions presented in this section only cover those articles that have been published in a peer reviewed journal. Preprinted articles are outside the scope of the current review discussion.

In [2] the analysis of confirmed cases of COVID-19 through a binary classification using artificial intelligence and regression analysis was investigated. In their study, the authors employed the binary classification modelling with group method of data handling type of neural network as one of the artificial intelligence methods of accurately predicting confirmed cases of the COVID-19 epidemic. The study chose the Hubei province in China for their model construction. For the input and output variables, some important factors such as maximum, minimum, and average daily temperature, city density, relative humidity, and wind speed, were considered as the input dataset, while the number of confirmed cases was selected as the output dataset for 30 days. Moreover, the outcome of the investigation revealed that the proposed binary classification model was able to provide a higher performance capacity in predicting the confirmed cases in the province. In addition, the analysis of the results also showed that certain weather conditions based on the input variables, namely relative humidity with an average of $77.9 \%$ had a positive impact on the confirmed cases and maximum daily temperature with an average of $15.4{ }^{\circ} \mathrm{C}$ had a negative impact on the confirmed cases. 
Mohammed, et al. [3] presented the application of two optimization metaheuristic techniques to enhance the predictive performance accuracy of the proposed adaptive neuro-fuzzy inference system that is used for estimating and forecasting the number of confirmed cases of novel coronavirus in the upcoming ten days based on previously confirmed cases that were recorded in China. The developed hybrid metaheuristic based adaptive neuro-fuzzy inference system comprised of Adaptive Neuro-Fuzzy Inference engine and two metaheuristic algorithms namely, flower pollination algorithm and salp swarm algorithm. The enhanced flower pollination algorithm was utilized by the author to train the neuro-fuzzy inference system by optimizing its parameters, while the salp swarm algorithm was incorporated as a local search method to enhance the quality of the solution obtained by the model. The results of the model implementation show that it has a high capability of predicting the number of confirmed cases within the projected ten days. It was further established that the hybrid system, when compared with other methods, obtained more superior performance accuracy in in terms of the following performance metrics: root mean square error, mean absolute error, mean absolute percentage error, root mean squared relative error, and coefficient of determination.

Ting, et al. [4] explored the potential application of four inter-related digital technologies combating the wide spread of the novel coronavirus. These technologies include the Internet of Things, big-data analytics, Artificial Intelligence and blockchain. The authors in their work [4] presented some valid reasons why the four aforementioned digital technologies can be employed to augment the already strained traditional based public-health strategies for tackling COVID-19. Some of the traditional based public healthcare strategies that have been put in place and are constantly being used across the globe include: (1) monitoring, surveillance, detection and prevention of COVID-19; and (2) mitigation of the impact to healthcare indirectly related to COVID-19. The authors further suggested that digital technologies can be helpful in the following ways. The Internet of Things technology can be used to provide a platform that allows public-health agencies access to data for monitoring the COVID-19 pandemic. The big data technology can be very useful in providing opportunities for performing modelling studies of viral activity and for guiding an individual country's healthcare policymakers to enhance preparation for the outbreak. The blockchain technology can be vital in the manufacturing and distribution of COVID-19 vaccines once they are available. Similarly, blockchain can be utilized to facilitate the distribution of patients' regular medication to the local pharmacy or patients' doorstep. The AI and deep learning technology can be used to enhance the detection and diagnosis of COVID-19. Further, the utilization of various AI-based triage systems could potentially alleviate the clinical load of physicians.

Vaishya, et al. [5] in their study highlighted the significant roles that some of the new technologies such as artificial intelligence, Internet of Things, Big Data and Machine Learning are likely to play in the fight against the new diseases and also the possible forecasting of any pandemics. The authors in [5] focused on presenting a brief review regarding the utilization of artificial intelligences platforms as a decisive technology to analyze, prepare us for prevention and fight against COVID-19 and any other similar pandemics. In their findings, seven significant application areas of artificial intelligence technology were identified for tackling the spread of COVID-19 disease. These areas as mentioned in [5] include: early detection and diagnosis of the infection, monitoring the treatment, projection of cases and mortality, development of drugs and vaccines, reducing the workload of healthcare workers, and prevention of the disease. Furthermore, the technology was also identified as having the capability to detect clusters of cases and predict the possible location of the virus spread through collecting and analyzing all previous data.

Leung and Leung [6] presented a discussion on the way forward in terms of crowdsourcing data to mitigate epidemics. The authors surveyed different and varied sources of possible line lists for COVID-19. The sources considered by the authors include data clearing houses or secondary repositories and official websites or social media accounts of various Health Commissions at the provincial and municipal levels in mainland China. Some of the main bottlenecks attributed to the process of crowdsourcing were linked to the rigorous tasks involved in carefully collating as much relevant data as possible, sifting through and verifying the data, extracting intelligence to forecast and inform outbreak strategies, and thereafter repeating this process in iterative cycles to monitor and evaluate progress [6]. However, a possible methodological breakthrough in alleviating these challenges would be to develop and validate algorithms for automated bots to search through cyberspaces of all sorts, by text mining and natural language processing to expedite these processes. Next, we present a brief discussion of some applications of CBR to healthcare with a specific focus on its utilization for analysis, prediction, diagnosis, and recommending treatment for patients. 
The CBR is an appropriate methodology to apply in the diagnosis and treatment of wide range of health issues. Research in CBR has grown to an extent, starting from the early exploration in the medical field by Koton [7], Bareiss [8] in the late 1980s and Gierl, et al. [9] in the late 1990s. However, there are still some associated shortcomings with the design and implementation of CBR, especially in the adaptation mechanism. Blanco, et al. [10] reported the results of a systematic review of CBR application to the health sector. In their work, the authors proposed some enhancement procedures that could be applied to overcome some of the limitations of CBR, which is focused on preparing the data to create association rules that help to reduce the number of cases and facilitate learning of adaptation rules.

CBR has equally received noticeable attention in the aspect of disease predictions and diagnosis. In [11], a hybrid implementation of neural networks and case-based reasoning was proposed for the prediction of chronic renal disease among the Colombian population. The neural network-based classifier which was trained with the demographic data and medical care information of two population groups was developed to predict whether a person is at risk of developing chronic kidney disease. The result of the classifier showed that about 3,494,516 people were identified as being at risk of developing chronic renal disease in Colombia, which in this case is $7 \%$ of the total population.

Benamina, et al. [12] proposed the integration of fuzzy logic and data mining technique to improve the response time and the accuracy of the retrieval step of case-based reasoning of similar cases. The Fuzzy CBR proposed in [12] is composed of two complementary parts, namely, the part of classification by fuzzy decision tree realized by Fispro and the part of case-based reasoning realized by the platform JColibri. The main function of fuzzy logic is to reduce the complexity of calculating the degree of similarity that can exist between diabetic patients who require different monitoring plans. The authors compared their results with some existing classification methods using accuracy as performance metrics. The experimental result that was generated by the proposed system revealed that the fuzzy decision tree is very effective in improving the accuracy for diabetes classification and hence improving the retrieval step of CBR reasoning.

Table 1 presents a concise summary of all published work on mathematical modeling, statistical modeling and simulation based literature on COVID-19 that appeared in Web of Science database. Each paper information is represented based on the article authors' details in column two, paper title in column three and reference in column four. Overall, sixteen publications were retrieved from the Web of Science database.

Table 1: Summary of all published related modeling and simulation based work on COVID-19 from Web of Science

\begin{tabular}{|c|c|c|c|}
\hline SN & Author & Title & ref \\
\hline 1 & $\begin{array}{l}\text { Rao, Arni S. R. Srinivasa; Krantz, Steven } \\
\text { G.; Kurien, Thomas; et al. }\end{array}$ & $\begin{array}{l}\text { Model-based retrospective estimates for COVID-19 or } \\
\text { coronavirus in India: continued efforts required to } \\
\text { contain the virus spread }\end{array}$ & {$[23]$} \\
\hline 2 & Buonomo, Bruno & $\begin{array}{l}\text { Effects of information-dependent vaccination behavior } \\
\text { on coronavirus outbreak: insights from a SIRI model }\end{array}$ & [24] \\
\hline 3 & $\begin{array}{l}\text { Kim, Soyoung; Kim, Yae Jean; Peck, Kyong } \\
\text { Ran; et al. }\end{array}$ & $\begin{array}{l}\text { School Opening Delay Effect on Transmission } \\
\text { Dynamics of Coronavirus Disease } 2019 \text { in Korea: } \\
\text { Based on Mathematical Modeling and Simulation Study }\end{array}$ & [25] \\
\hline 4 & $\begin{array}{l}\text { Hellewell, Joel; Abbott, Sam; Gimma, Amy; } \\
\text { et al. }\end{array}$ & $\begin{array}{l}\text { Feasibility of controlling COVID-19 outbreaks by } \\
\text { isolation of cases and contacts }\end{array}$ & [26] \\
\hline 5 & Jia, Jiwei; Ding, Jian; Liu, Siyu; et al. & $\begin{array}{l}\text { Modeling the control of COVID-19: Impact of policy } \\
\text { interventions and meteorological factors }\end{array}$ & [27] \\
\hline 6 & Choi, Sunhwa; Ki, Moran & $\begin{array}{l}\text { Estimating the reproductive number and the outbreak } \\
\text { size of COVID-19 in Korea }\end{array}$ & [28] \\
\hline 7 & Huang, Rui; Liu, Miao; Ding, Yongmei & $\begin{array}{l}\text { Spatial-temporal distribution of COVID-19 in China } \\
\text { and its prediction: A data-driven modeling analysis }\end{array}$ & [29] \\
\hline 8 & $\begin{array}{l}\text { Gostic, Katelyn; Gomez, Ana C. R.; } \\
\text { Mummah, Riley O.; et al. }\end{array}$ & $\begin{array}{l}\text { Estimated effectiveness of symptom and risk screening } \\
\text { to prevent the spread of COVID-19 }\end{array}$ & [30] \\
\hline 9 & Wang, Chuanyi; Cheng, Zhe; Yue, Xiao- & & [31] \\
\hline
\end{tabular}


Guang; et al.

10 Roosa, Kimberlyn; Lee, Yiseul; Luo, Ruiyan; et al.

11 Yang, Shu; Cao, Peihua; Du, Peipei; et al.

12 Jiang, Xiangao; Coffee, Megan; Bari, Anasse; et al.

13 Yin, Fulian; Lv, Jiahui; Zhang, Xiaojian; et al.

14

Zhou, Weike; Wang, Aili; Xia, Fan; et al.

15

Yang, Chayu; Wang, Jin

16

Rong, Xinmiao; Yang, Liu; Chu, Huidi; et al.

17 Hou, Can, Jiaxin Chen, Yaqing Zhou, Lei Hua, Jinxia Yuan, Shu He, Yi Guo et al.

18 Prem, Kiesha, Yang Liu, Timothy W. Russell, Adam J. Kucharski, Rosalind M. Eggo, Nicholas Davies, Stefan Flasche et al.

19 Ho, Dean

20 Shi, Feng, Jun Wang, Jun Shi, Ziyan Wu, Qian Wang, Zhenyu Tang, Kelei He, Yinghuan Shi, and Dinggang Shen

21 Kim, Donghyun, Soyoung Hong, Sungwoo Choi, and Taeseon Yoon.
Risk Management of COVID-19 by Universities in China

Short-term Forecasts of the COVID-19 Epidemic in Guangdong and Zhejiang, China: February 13-23, 2020

Early estimation of the case fatality rate of COVID-19 in mainland China: a data-driven analysis

Towards an Artificial Intelligence Framework for DataDriven Prediction of Coronavirus Clinical Severity

COVID-19 information propagation dynamics in the Chinese Sina-microblog

Effects of media reporting on mitigating spread of COVID-19 in the early phase of the outbreak

A mathematical model for the novel coronavirus epidemic in Wuhan, China

Effect of delay in diagnosis on transmission of COVID19

The effectiveness of the quarantine of Wuhan city against the Corona Virus Disease 2019 (COVID-19): well-mixed SEIR model analysis

The effect of control strategies to reduce social mixing on outcomes of the COVID-19 epidemic in Wuhan, China: a modelling study

Addressing COVID-19 Drug Development with

Artificial Intelligence.

Review of artificial intelligence techniques in imaging data acquisition, segmentation and diagnosis for Covid19

Analysis of transmission route of MERS coronavirus using decision tree and Apriori algorithm

\subsection{Bibliometric Analysis of COVID-19 Research Activities}

In this paper the VOSviewer software was used to present a bibliometric analysis of the existing literature on COVID-19. VOSviewer software is a tool for constructing and visualizing bibliometric maps of items, for example journals, researchers, or individual publications. These maps can be constructed based on citation, bibliographic coupling, co-citation, or co-authorship relations. Further, the bibliometric analysis software also offers text mining functionality that can be used to construct and visualize co-occurrence networks of important terms extracted from a body of scientific literature (see www.vosviewer.com). In this section, we present the bibliographic coupling among different article items on COVID-19. The link between the items on the constructed map corresponds to the weight between them either in terms of number of publications, common references or co-citations. These items may belong to a group or cluster. In the visualization, the items within the same cluster are marked with same color. Colors in this case indicate the cluster to which a journal was assigned by the clustering technique implemented by the VOSviewer software. The items may be represented by the circular node and its size may vary depending on the weight of the article. We only used 488 publications with the key word "Coronavirus COVID-19" that were retrieved from the Web of Science database for the bibliometric analysis presented in this paper. No consideration was given to articles from other sources, as most of these publications appears not to have been peer reviewed or are available online in the form of preprint publications. Similar bibliometric analyses have been reported in literature as presented by Chahrour, et al. [44], Hossain [45] and Lou, et al. [46]. However, these existing analyses differ from the current bibliometric analysis in the sense that the current analysis result focused more on the application of AI techniques to combat COVID-19 pandemic as opposed to the various literature reporting general medical practices on COVID-19. 
The bibliographic coupling between the top 25 authors is shown in Figure 1. The two clusters, namely red and green, correspond with all the authors working on similar research fields "COVID-19" and citing the same source in their reference listings. The similarity in cluster color for the authors also implies that the degree of overlap between the reference lists of publications of these authors is greater. Shown in Figure 1 are names which are visible; there are possibly other names which are not represented in the constructed map.

Figure 2 shows the bibliographic coupling of the top most productive countries. Here, bibliographic coupling indicates that there is a more common reference list in the papers published by these countries. There are clearly five clusters, which are represented by five colors, namely, the blue color with China and USA having the highest strength in terms of contribution, green color with England and Italy having the highest strength, red color with India and Germany having the highest strength, lemon color with Japan as the highest contributor and purple color with Switzerland as the only contributing country. The link between the blue and green clusters can be seen to be thicker compared to the link between the blue and red clusters or between the blue and purple clusters. The thickness of the link simply depicts the degree of intersection of the literature work between the different locations or countries.

Figure 3 shows the bibliographic coupling of the network of collaborating institutions that were affiliated with at least four documents on COVID-19 research. From the map construction and analysis, of the 949 institutions, 28 meet the thresholds of collaborating institutions, including Huazhong University of Science and Technology, Wuhan University, Capital Medical University, Chinese Academy of Medical Sciences, University of Hong Kong, Sun YatSen University, and Fudan University. There are four clusters identified by blue, red, green and lemon colors. It can be observed that the institutions that fall under the same clusters appear to have similar literature background or worked on related research fields.

Bibliographic coupling between the journals implies that the papers published in these journals have more common reference lists. Clearly there are three clusters that are depicted on the map with red, blue, and green colors. The links with highest strength occurs between the Journal of Medical Virology and Cureus Journal of Medical Science. This is closely followed by the links between Medical Virology and Cureus and Eurosurveillance Journal, Archive of Academic Emergency Medicine, Journal of Korean Medical Science and Lancet. The medical science monitor and archive Iranian medicine form the weakest networks of cluster. Figure 4 below clearly illustrates the bibliographic coupling between the considered journals. 


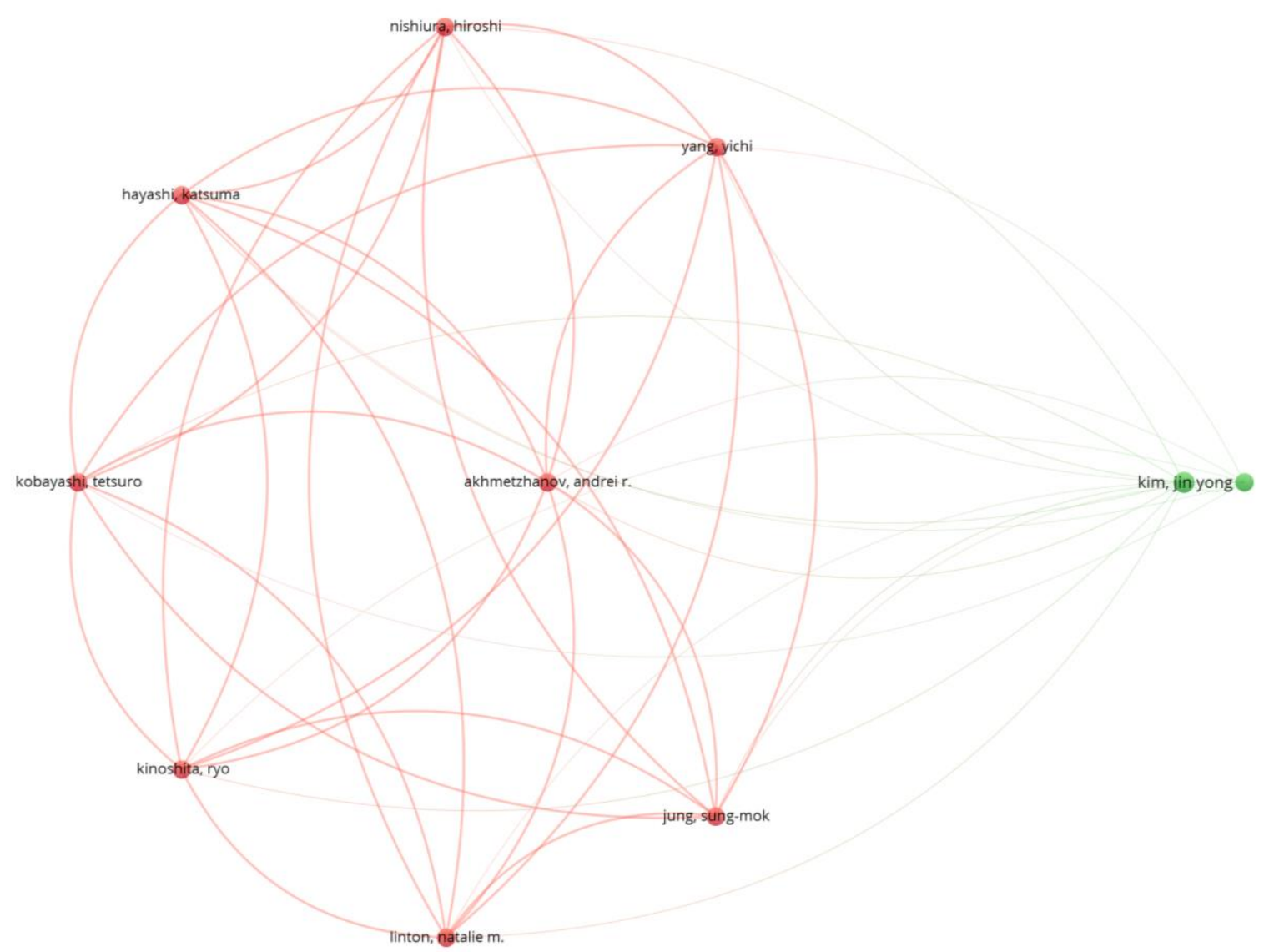

Figure 1: Bibliographic coupling among the authors 


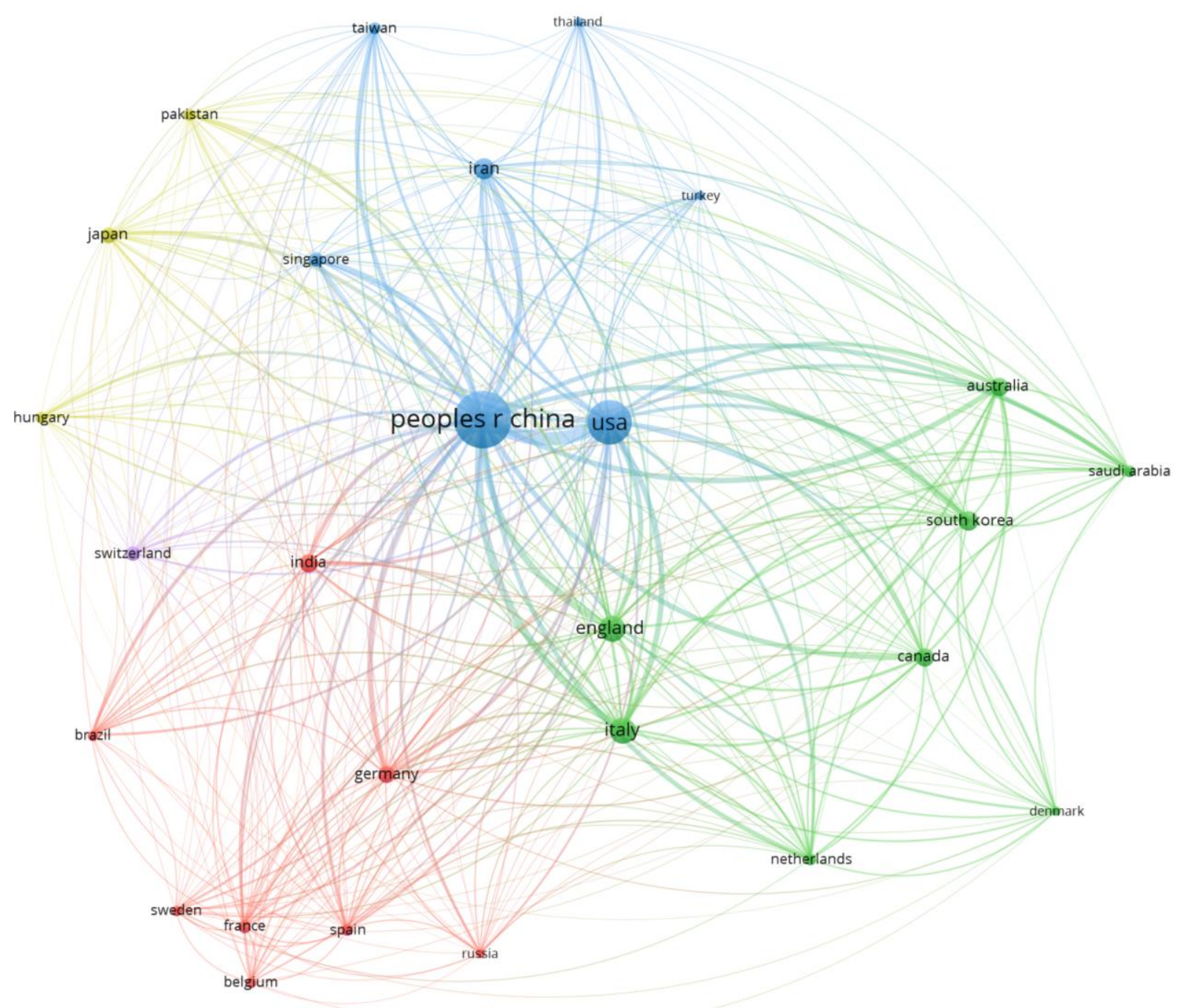

Figure 2: Bibliographic coupling among the countries 


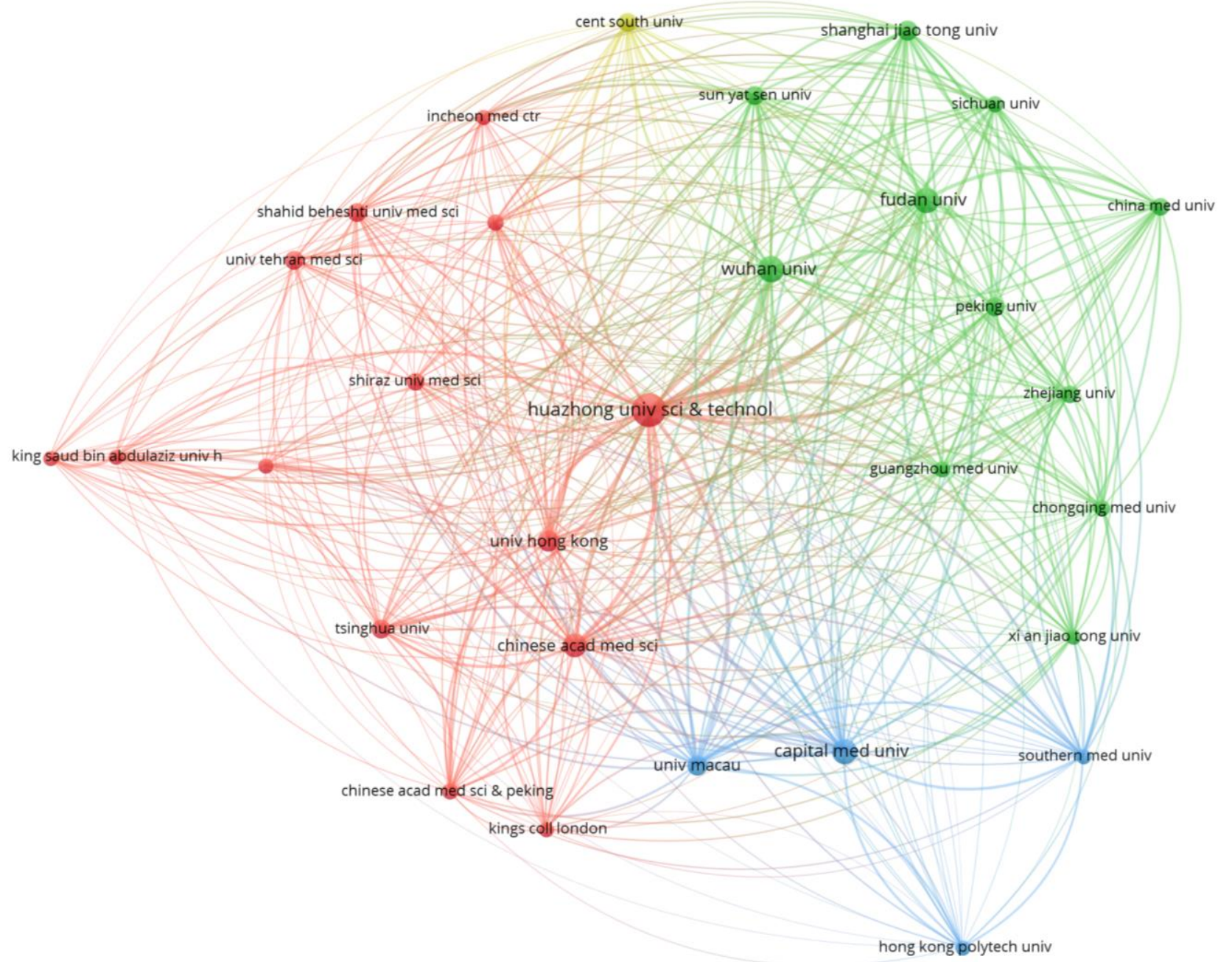

Figure 3: Bibliographic coupling among institutions 


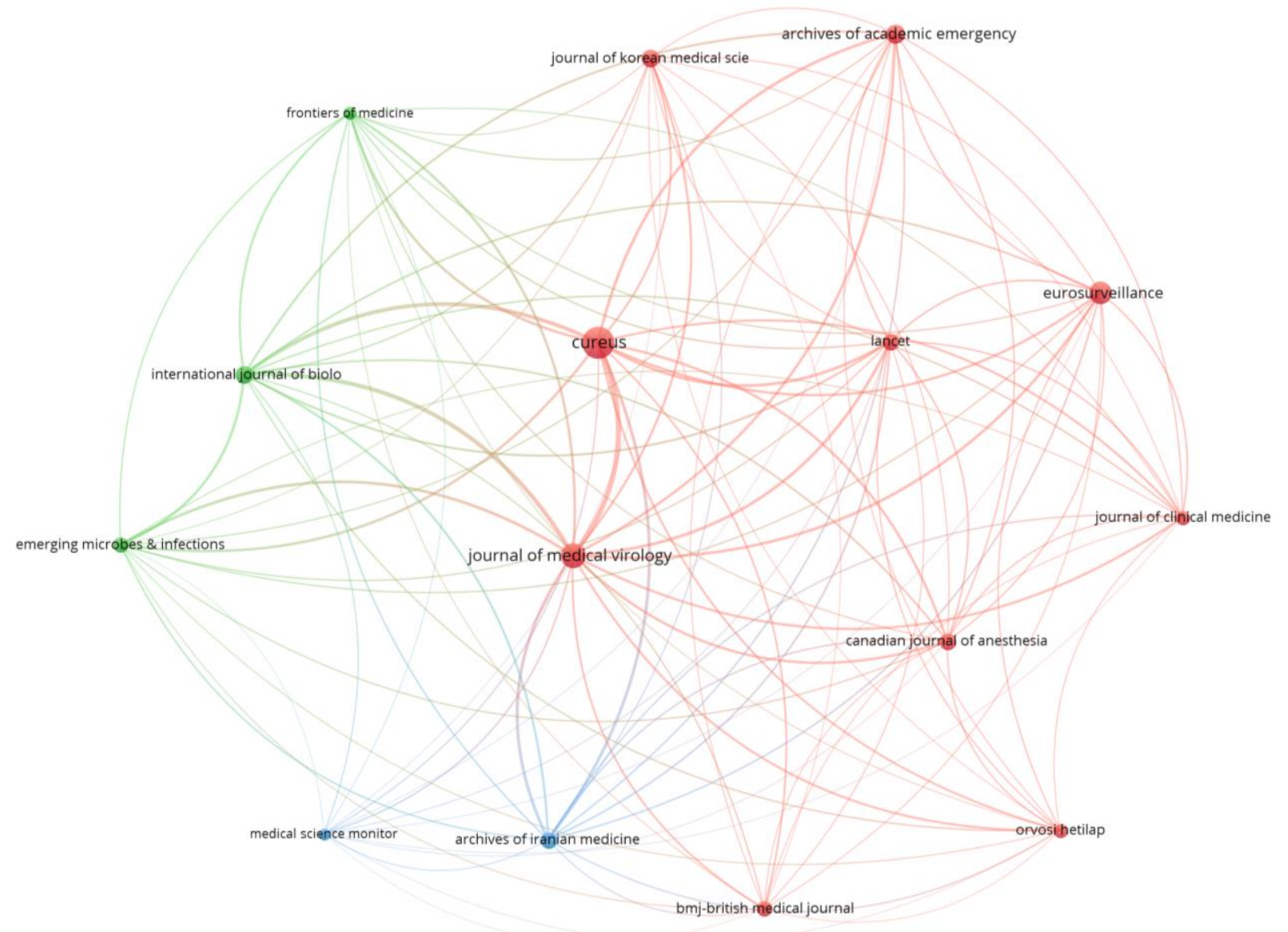

Figure 4: Bibliographic coupling among the Journals

Figure 5 illustrates the co-authors and authors map visualization. The essence of this analysis is to produce the visualization of all the major authors publishing together or working on similar research fields. Here, the analysis type is co-authorship and the unit of analysis is authors. The threshold of minimum number of papers by an author is 25. From the network construction and analysis, of 2,381 authors, only 9 authors meet the thresholds. However, the largest set of connected entities consists of only 8 authors, whose visual representation is depicted in Figure 5. From the figure, there is only one cluster denoted by red color. The connected link depicts that these authors have collaborated on the same project or worked on the same research with a similar focus. The thickness of the link between these three authors indicates more common publications.

Figure 6 illustrates the citation analysis among authors' institutions. There are six clusters represented with different colors. The red colored cluster has the highest number of authors citations from two institutions, namely, Huazhong University of Science and Technology and Wuhan University. Figure 7 shows the bibliometric analyses of author citations by journal sources. A link between two journal sources indicates the citation connectivity between the two source. Moreover, the connected links between say Cureus journal and Lancet in Figure 7 clearly reveals that either a publication from Cureus has cited another publication that was published in Lancet journal or otherwise. The thickness and link strength signifies more numbers of citation among the clusters. Therefore, among the different clusters identified in the analysis, Medical Virology and Cureus journals are the top cited source by publication from other journal sources. 


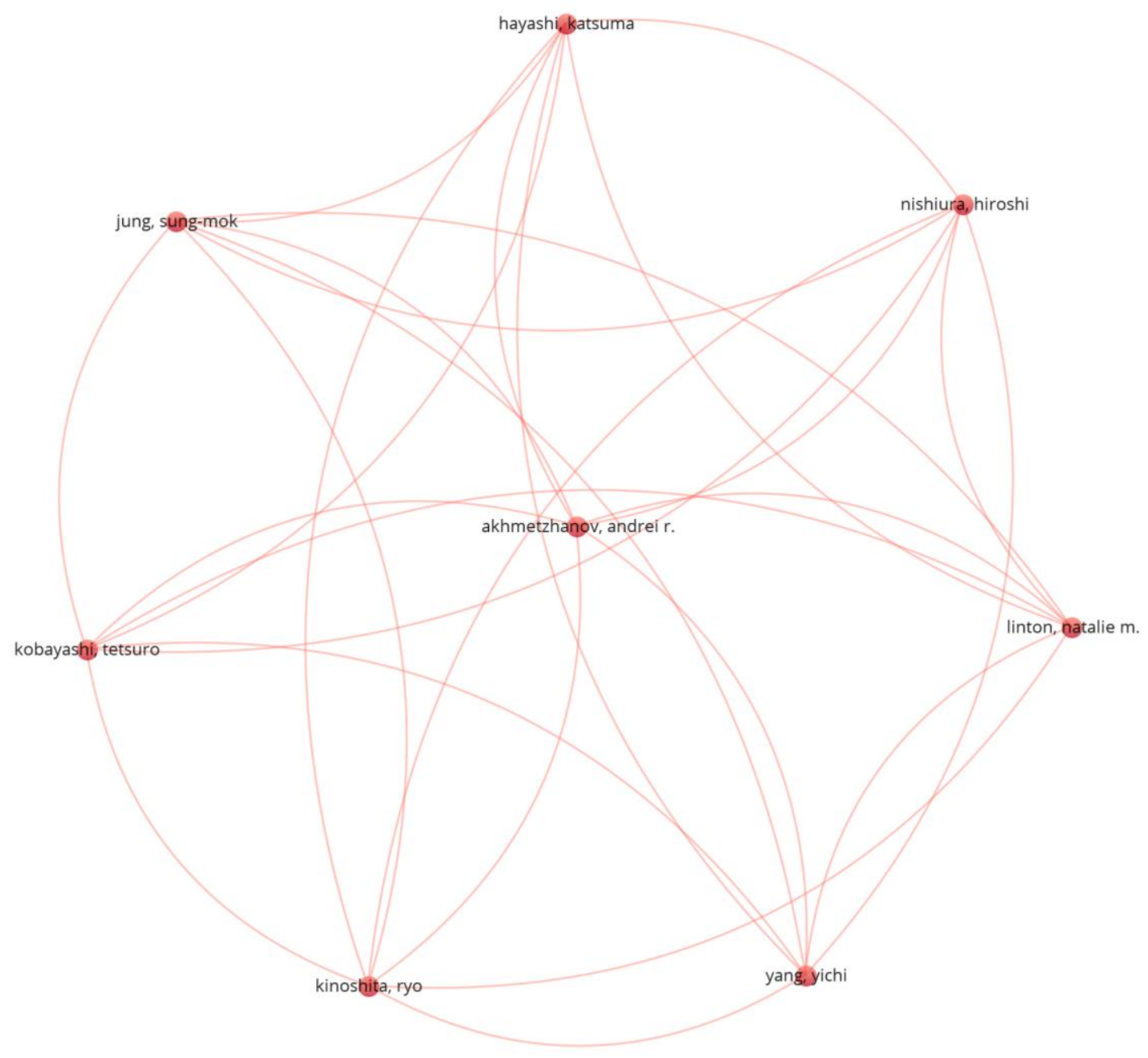

Figure 5: Co-authorship and Authors' analysis 


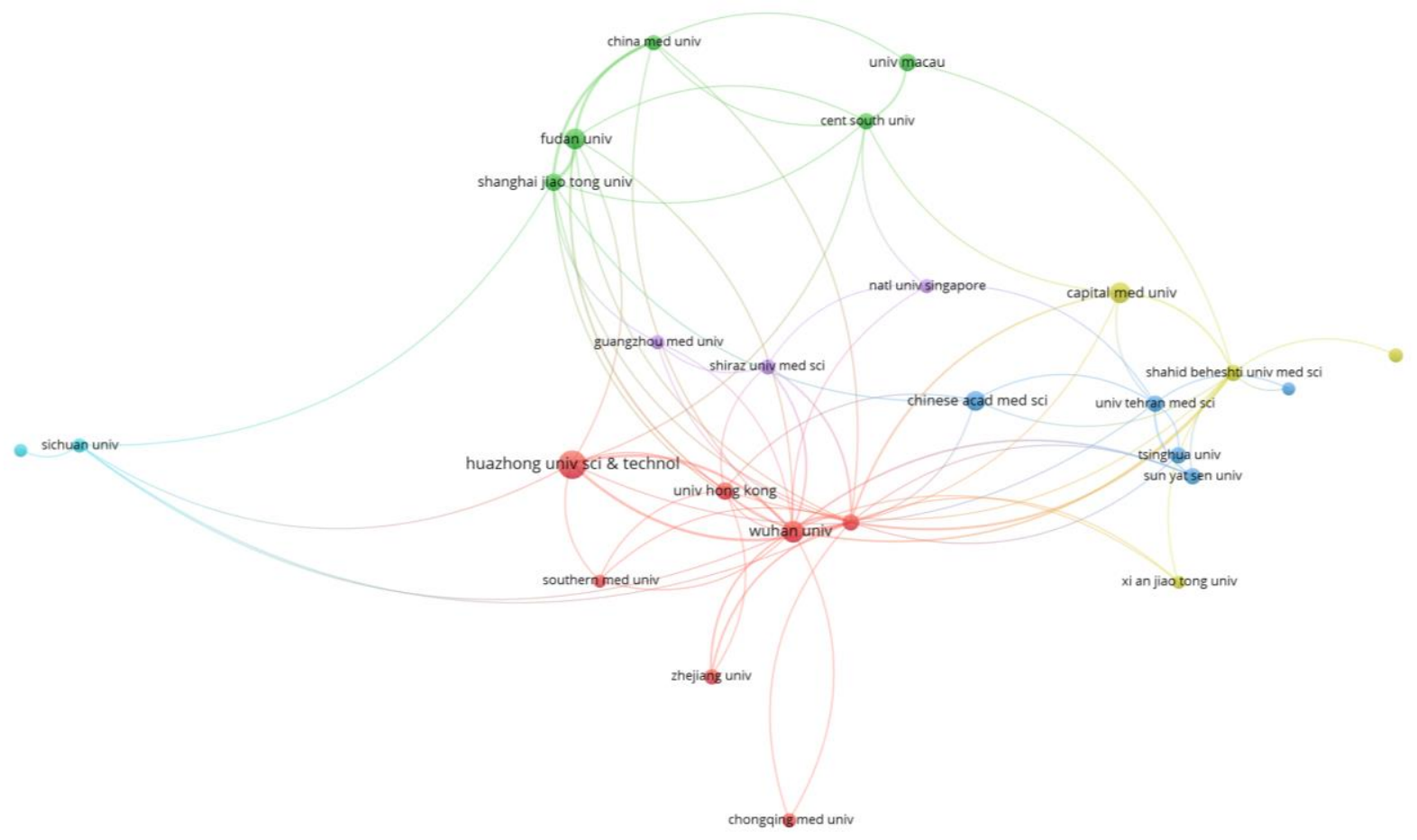

Figure 6: Author citation by institution

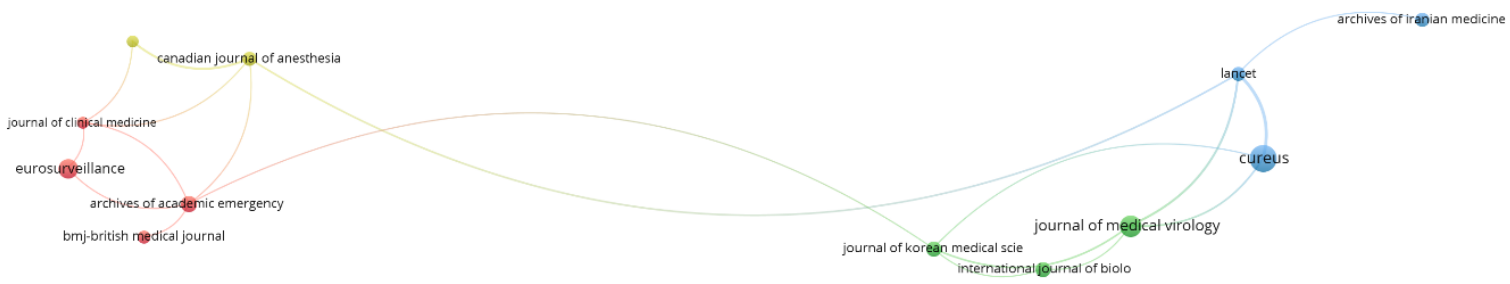

Figure 7: Author citation by journal source.

\subsection{Proposed Approach}

A detail presentation of the methods adopted and adapted in this study is covered in this section: an overview of the entire approach, feature extraction using a natural language processing technique, formalism of cases in the proposed case-based reasoning (CBR) method, and lastly the CBR engine.

\subsection{An Overview of the Approach}

The proposed NLP-Ontology-CBR method accepts a text-based patient file as input for processing of status of the case. Figure 8 presents an illustration of all procedures applied to the inputs denoted by a case file. The case file is passed as input into an NLP Text-2-Features module. This module leverages on some natural language processing operations to synthetically and semantically extract tokens from the case file. The extracted case features are further passed to the domain-based feature extraction component which maps each extract feature at the previous layer to domain-based features. The extracted and mapped features are formalized using description logic (DL) based on a knowledge representation format to allow for efficient computational operations in the CBR engine. Finally, the formalized features are passed on to the CBR-engine as a new case (nc) that support the application of the reasoning paradigm of CBR. 
The pipeline of information flow and processing described in Figure 8 was therefore adapted to detect the case of positive COVID-19 patient from early stage to the advance stage. A further discussion in the following subsections details the components of the framework. 


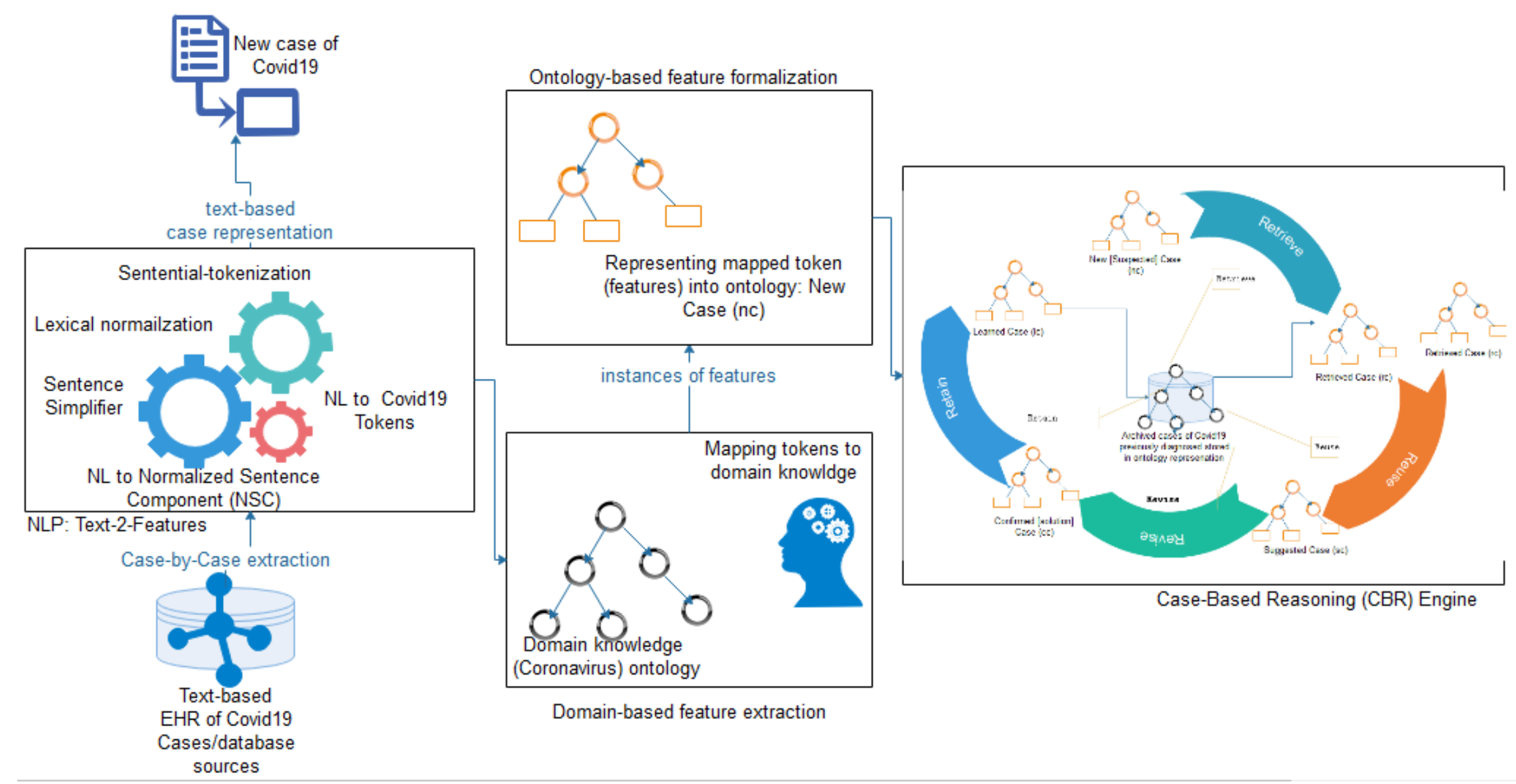

Figure 8: An overview of the proposed framework using case-based reasoning (CBR) model to classify new cases of coronavirus (COVID-19) as either positive or negative 


\subsection{The NLP Method for Feature Extraction}

The field of natural language processing technique is a very interesting and relevant aspect of artificial intelligence (AI) with a wide range of applications to medicine and even the large number of text-based documents on the internet. Moreover, electronic health record (EHR) systems are now pervasive and are provided as services to other automated healthcare delivery systems. The NLP method for feature extraction described in this section adopts some components and algorithms from Dasgupta, et. al. [47]. The ontology learning method is widely used for mining information from natural language text and generating an ontology representation of the mined data. Such ontology representation is to provide formal expressivity and a platform for reasoning with such NP-text document. Although this study assumes a similar procedure, we implemented a skeletal of the entire procedure.

Figure 9 shows the modified model of a patient text-based medical record natural language processing (NLP) and features extraction pipeline. The model is called a pipeline because of its approach of processing raw file-based text (in English language) through different procedures which eventually yields the feature (Covid-Fs) for further processing in the CBR-engine.

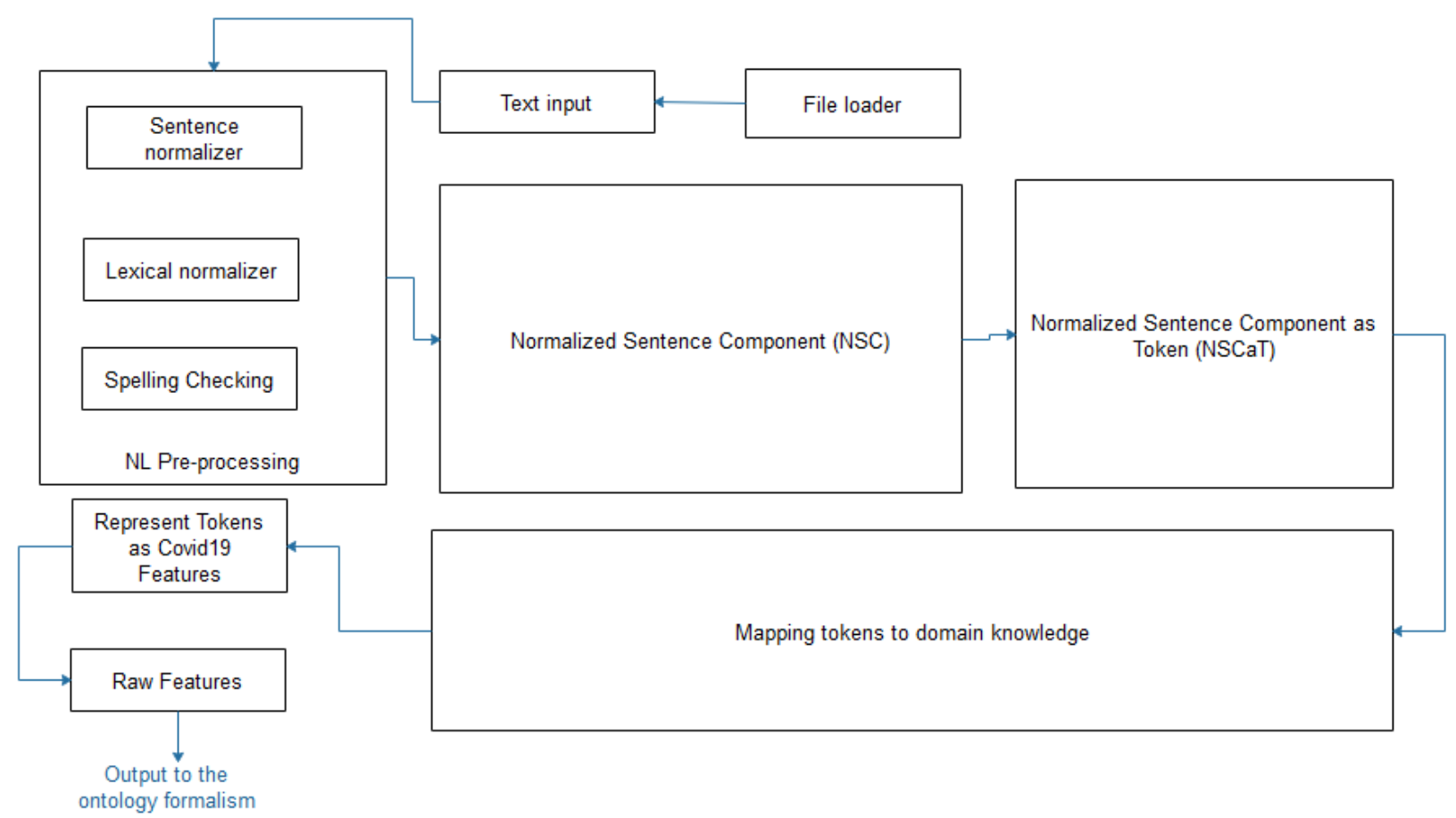

Figure 9: A patient text-based medical record natural language processing (NLP) and feature extraction architectural pipeline

The following is a breakdown of the components of the NLP processing pipeline as shown in Figure 9:
a. File loader and Text input (FLTI)
b. NL Pre-processing: Spelling checking, Lexical normalizer, Sentence normalizer
c. Normalized Sentence Component (NSC)
d. Normalized Sentence Component as Token (NSCaT)
e. Mapping tokens to domain knowledge (MTDK)
f. Represent Tokens as COVID-19 Features (RTCF)
g. Raw Features Buffer (RFB)

File Loader and Text Input (FLTI): The FLTI is a very simple component with support for file format and safety authentication, file loading and text-content unloaded into a buffer.

NL Pre-processing (NL-P): The second component consists of other sub-modules named Spelling Checking, Lexical normalizer, and Sentence normalizer which does a pre-processing of the buffered text in FLTI layer. 
Generally, the NL-P is aimed at carrying out operations like spell-corrector, tokenization, sentence boundary detector, text singularizer, POS-tagger, co-reference resolver, and named-entity recognizer (NER) by leveraging on Stanford coreNLP toolkit [48]. Our approach of applying NL-P to the buffered text in FLTI was to allow the spellcorrector to scan through the complete buffer and correct wrongly spelt words and furthermore to allow for efficient and intelligent mining of features from the buffered text - the improved output of FLTI. This was then converted into a token of sentential forms (SF) in list format and then sorted according to their appearance in the original document. In each SFs, we attempted to normalize each plural form of its constituents into a singular form through the use of a singularizer. These SFs were extracted from buffered text using sentence boundary detector and annotated with POS-tagging, and the SFs were preserved in an orderly manner to sustain the semantics of health records. Meanwhile, due to the translation task of the raw text to ontology format, we further employed NER models to identify and mark entities and thereafter their instances which form the elements of taxonomy-box (TBox) and assertion box (Abox) respectively in the resulting ontology. Once the SFs had been pre-processed, we applied them to the next sub-module named lexical normalizer $(\mathrm{LN})$. The use of $\mathrm{LN}$ in our study is simply for identification of quantifiers and special symbols (like $>,<,=,+,-$, and other medical related symbols which may hold meaning in the usage) of subject/objects appearing in the SFs. Our approach in LN allows for such quantifiers/numeric representations and symbols to be normalized into normal forms supportive of the token-to-feature translation in RTCF component of Figure 9. The role of applying the sentence normalizer (SN) is to ensure that very difficult sentences are broken down to simple forms so that an element of $\mathrm{SFs}$, say $\mathrm{sf}_{\mathrm{i}}$, is normalized into simpler forms assuming the template of the NSC component to be discussed later. Hence, the resulting simplified sentences of sf $\mathrm{i}_{\mathrm{i}}$ replace it in SFs.

Normalized Sentence Component (NSC): Based on the structural formation of a sentence in English language, a particular template or syntax was described by Dasgupta, et. al. [47] in their study. We adopted two of the templates, namely the simple and complex sentences as listed in the following:

\section{$\underline{\mathbf{Q}_{1}} \underline{\mathbf{M}_{1}} * \mathbf{S}$ is-a ${\underline{Q_{2}}}_{\underline{\mathbf{M}_{2}}}^{*} \mathbf{O}$ \\ $\underline{Q}_{1} \underline{M}_{1}^{*} S C_{1}$ IS-A $\underline{Q}_{2} \underline{M}_{2}^{*} O_{1} C_{2}$ IS-A $\underline{Q}_{3} \underline{M}_{3}^{*} O_{2}$}

Q Under-lined notation indicates optional component with at most 1 occurrence in the template e.g. quantification

M* Under-lined notation with asterisk (*) indicates 0 or more consecutive occurrences in the template e.g. adjectives.

Q1 Subject quantifier that includes lexical variations of the set: a, an, the, some, all.

$\mathbf{Q}_{2} \quad$ Object quantifier that includes lexical variations of the set: the, some, all.

Q3 Object quantifier that includes lexical variations of the set: the, some, all.

M Subject/object/verb modifier; value is restricted to the set: Noun, Adjective, Adverb, Numerical, and Gerund

S Subject; value is restricted to the set: fNN, NNP25, JJ, RB, VBGg

O Object; value is restricted to the set: fNN, NNP, JJ, RB, VBGg

IS-A Denotes all possible lexical variations.

$\mathbf{C l}_{\mathbf{1}}$ and $\mathbf{C l}_{\mathbf{2}}$ signifies IS-A clausal token and all its variations 'which', 'who', 'whose', 'whom', 'that'.

Finally, we ensured that all the sentences in SFs were adapted to the template described above and then we applied their Template-Fitting algorithm to all elements of SFs.

Normalized Sentence Component as Token (NSCaT): The CBR-engine to be described in Sub-section 3.4 does not expect input in sentential format but tokenized features which maintains its sentence form syntax and semantics. Therefore, each $s_{i}$ in SFs are further tokenized into a list of raw (un-normalized features) tokens in the form of $t_{i j}$ such that $\boldsymbol{i}$ represents the position of the sentence in SFs and $\boldsymbol{j}$ represents the position of the token in the $\mathrm{sf}_{\mathrm{i}}$ of SFs that is being processed. The output of NSCaT is therefore an irregular 2D array of raw tokens.

Mapping Tokens to Domain Knowledge (MTDK): We assumed that not all the tokens from NSCaT are correctly represented based on the domain knowledge. As a result, we proposed a MTDK layer which was aimed at mapping each token in the NSCaT to its correct recognized name in the domain. We relied strongly on Wordnet (WordNet) 
and the domain-based lexicon model in this study as shown in Figure 10. The role of the Wordnet lexicon is to generate all likely synonyms of each $t_{i j}$ in NSCat. This therefore means that each $t_{i j}$ indexes into a sub-array of its synonyms. Thereafter, our mapping algorithm aligns each $t_{i j}$ to its respective sub-array.

Represent Tokens as COVID-19 Features (RTCF): The output of MTDK is further refined to assume the standard feature categorization and typing as listed in Table 2. The implication of this is that we attempted to extract known features of COVID-19 from the output of MTDK and assigned their values as illustrated in Figure 11.

Raw Features Buffer (RFB): This last component simply buffers the output of raw features collected from previous layers. The RFs buffered in RFB are then translated into ontology formalism described in Section 4.2.

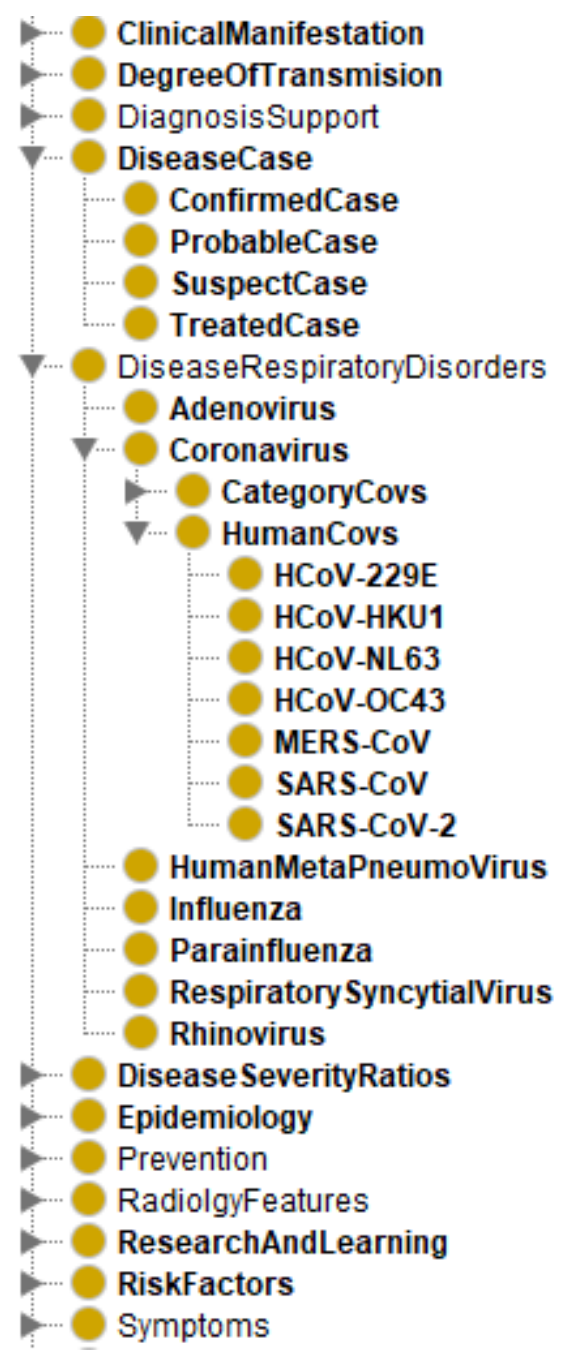

Figure 10: A lexicon of terminologies representing domain knowledge of COVID-19 in addition to symptoms, treatment, epidemiology, disease case status, and other relevant concepts in the domain

The features described in Table 2 were based on recent studies on COVID-19 that were discussed by Michelen, et al. [49] and Yang, et al. [33].

Table 2: A summary of categorization of coronavirus clinical-based features to be extracted by the domain-based feature extractor

\begin{tabular}{|l|lll}
\hline Feature category & Feature Name & Description of feature & $\begin{array}{l}\text { Feature } \\
\text { calibration }\end{array}$ \\
\hline
\end{tabular}




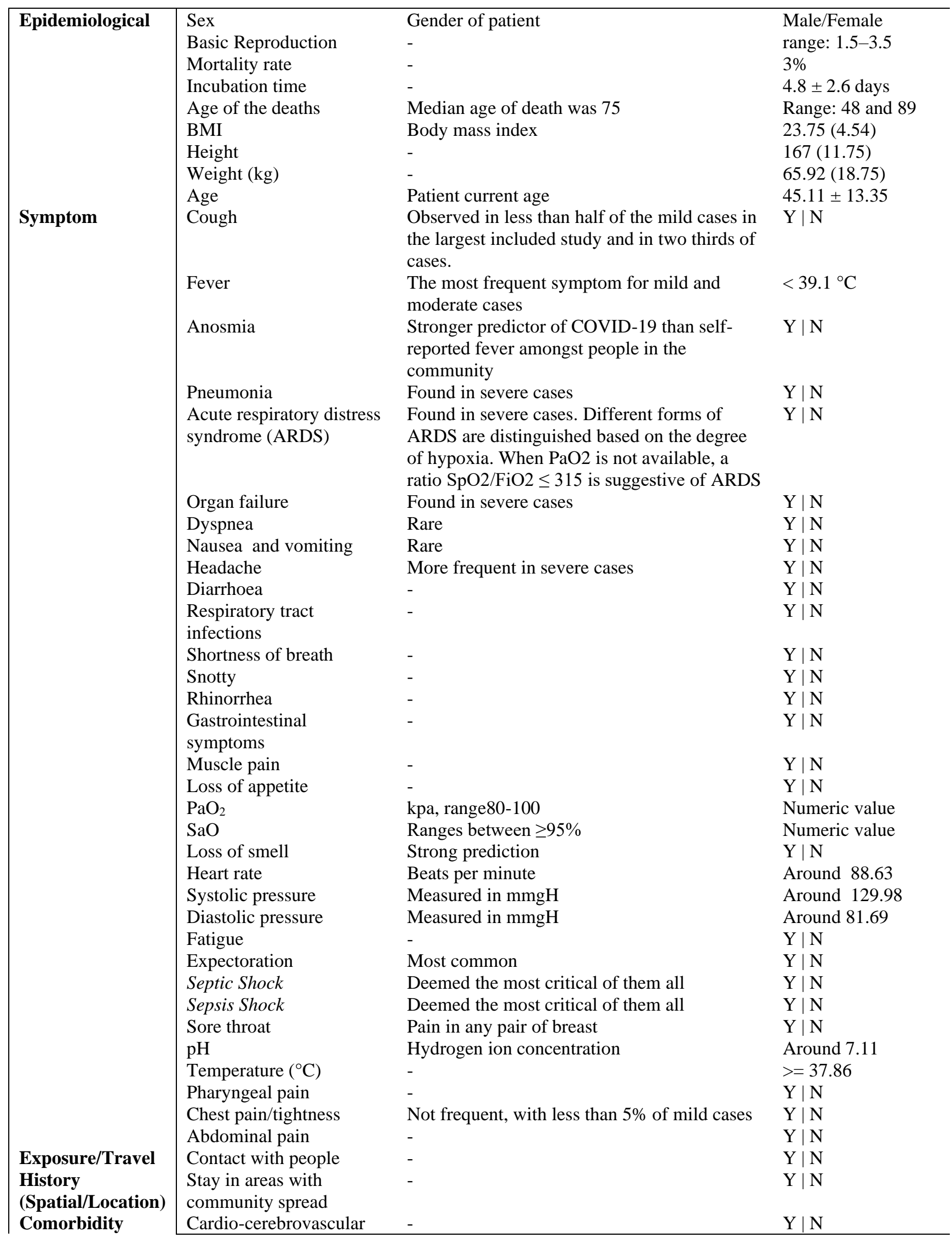




\begin{tabular}{|c|c|c|c|}
\hline \multirow{6}{*}{ (diseases) } & Digestive system & - & \begin{tabular}{l|l}
$\mathrm{Y}$ & $\mathrm{N}$ \\
$\mathrm{c}$
\end{tabular} \\
\hline & Endocrine diseases & - & $\mathrm{Y} \mid \mathrm{N}$ \\
\hline & Runny nose & - & $\mathrm{Y} \mid \mathrm{N}$ \\
\hline & Malignant tumor & - & $\mathrm{Y} \mid \mathrm{N}$ \\
\hline & Neural system & - & $\mathrm{Y} \mid \mathrm{N}$ \\
\hline & Respiratory system & - & $\mathrm{Y} \mid \mathrm{N}$ \\
\hline \multirow[t]{21}{*}{ Laboratory Tests } & neutrophil $\left(\times 10^{9}\right.$ per L) & - & range $1.8-6.3$ \\
\hline & Leucocyte $\left(\times 10^{9}\right.$ per L) & - & range $3.5-9.5$ \\
\hline & Lymphocyte $\left(\times 10^{9}\right.$ per $\left.\mathrm{L}\right)$ & - & range $1.1-3.2$ \\
\hline & platelet $\left(\times 10^{9}\right.$ per $\left.\mathrm{L}\right)$ & - & range $125-350$ \\
\hline & Blood coagulation & - & \\
\hline & $\begin{array}{l}\text { Active partial thrombin } \\
\text { time }\end{array}$ & - & range $22-36$ \\
\hline & Prothrombin time & - & range $10-13.5$ \\
\hline & D-dimer & - & range $<0.55$ \\
\hline & albumin & - & range $35-57$ \\
\hline & ALT (IU/L) & - & range $0-64$ \\
\hline & AST ((IU/L) & - & range $8-40$ \\
\hline & Total bilirubin $(\mu \mathrm{mol} / \mathrm{L})$ & - & range $4.7-24$ \\
\hline & Urea nitrogen $(\mathrm{mmol} / \mathrm{L})$ & - & range2.6-7.5 \\
\hline & Creatinine $(\mu \mathrm{mol} / \mathrm{L})$ & - & range $41-73$ \\
\hline & $\mathrm{CK}(\mathrm{mmol} / \mathrm{L})$ & - & range $40-200$ \\
\hline & LDH (U/L) & - & range $12-250$ \\
\hline & Serum Lactate $(\mathrm{mmol} / \mathrm{L})$ & - & range $>2$ \\
\hline & Glucose $(\mathrm{mmol} / \mathrm{L})$ & - & range3.9-6.1 \\
\hline & Coagulopathy & - & $\mathrm{Y} \mid \mathrm{N}$ \\
\hline & C-reactive protein (mg/L) & Infection-associated & range $0.0-6.0$ \\
\hline & Procalcitonin & Elevation to evidence of COVID-19 & - \\
\hline \multirow[t]{9}{*}{ Treatment } & Oxygen therapy & - & $\mathrm{Y} \mid \mathrm{N}$ \\
\hline & Antifungal treatment & - & $\mathrm{Y} \mid \mathrm{N}$ \\
\hline & Antiviral treatment & - & $\mathrm{Y} \mid \mathrm{N}$ \\
\hline & $\begin{array}{l}\text { Extracorporeal membrane } \\
\text { oxygenator (EMO) }\end{array}$ & - & $\mathrm{Y} \mid \mathrm{N}$ \\
\hline & Glucocorticoids & - & $\mathrm{Y} \mid \mathrm{N}$ \\
\hline & Antibiotic treatment & - & $\mathrm{Y} \mid \mathrm{N}$ \\
\hline & Intensive care unit (ICU) & - & $\mathrm{Y} \mid \mathrm{N}$ \\
\hline & $\begin{array}{l}\text { Noninvasive ventilation } \\
\text { (NIV) }\end{array}$ & - & $\mathrm{Y} \mid \mathrm{N}$ \\
\hline & $\begin{array}{l}\text { Invasive mechanical } \\
\text { ventilation (IMV) }\end{array}$ & - & $\mathrm{Y} \mid \mathrm{N}$ \\
\hline \multirow[t]{8}{*}{ Radiological } & Pulmonary infiltration & - & $\mathrm{Y} \mid \mathrm{N}$ \\
\hline & Air bronchogram & - & $\mathrm{Y} \mid \mathrm{N}$ \\
\hline & Centrilobular nodules & - & $\mathrm{Y} \mid \mathrm{N}$ \\
\hline & Tree-in-bud & - & $\mathrm{Y} \mid \mathrm{N}$ \\
\hline & Reticular pattern & - & $\mathrm{Y} \mid \mathrm{N}$ \\
\hline & Subpleural linear opacity & - & $\mathrm{Y} \mid \mathrm{N}$ \\
\hline & Bronchial dilatation & - & $\mathrm{Y} \mid \mathrm{N}$ \\
\hline & Cystic change & - & $\mathrm{Y} \mid \mathrm{N}$ \\
\hline
\end{tabular}




\begin{tabular}{llc}
\hline Lymphadenopathy & - & $\mathrm{Y} \mid \mathrm{N}$ \\
Pleural effusion & - & $\mathrm{Y} \mid \mathrm{N}$
\end{tabular}

\subsection{Ontology-based Formalization of Extracted Features}

In this stage of our proposed CBR-framework, we processed the raw features buffered in the RBF component of Figure 9 into ontology formalism. Recall that the proposed framework relies on the CBR paradigm to reasoning over the cases presented to it. Hence, each case was modeled using a formalism supporting computational reasoning operation. Figure 11 demonstrates an illustration of a case denoted by Case N. We assumed that based on clinical protocols of COVID-19, a case representation must have a relationship to Diagnosis Case (Suspected, Confirmed, Presumed status); Symptoms (as listed in Table 1); Epidemiology (as listed in Table 1); Radiology/Laboratory manifestations (as listed in Table 1); Clinical Diagnosis (Mild, Acute, Severe); and Treatment (as listed in Table 1). Each case of COVID-19 extracted by the NLP pipeline described in Figure 9 was formalized into this structure as illustrated in Figure 11. The Diagnosis Case entity assumes a 1-1 relationship with every case; Symptoms however presents with a one-many 1 to many (1-M) relationship for each case; also, the Epidemiology entity allows each case to manifest one-many (1-M) relationship; the Radiology/Laboratory manifestations entity also presents each case in a one-many (1-M) relationship given the number of lab tests and radiological operations that might be exercised for each case; Clinical Diagnosis, however, allows for one-one (1-1) relationship due to the fact that a case can only assume one of the states listed in clinical diagnoses; and finally, the Treatment entity allows for one-many (1-M) because one case may respond to one or more treatment/therapy administered to it.
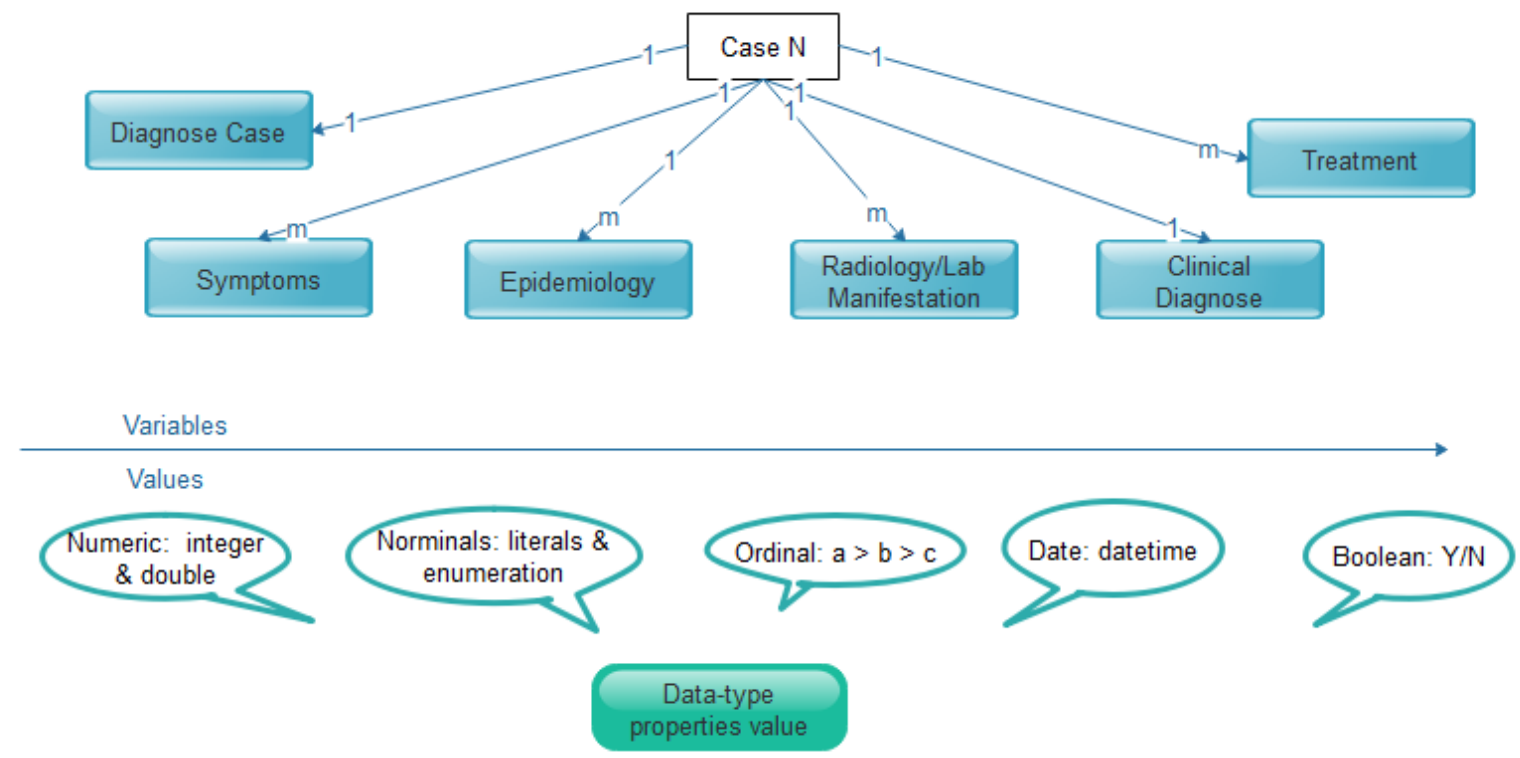

Figure 11: A formal representation tokens (features) of a new case (nc) of coronavirus (COVID-19)

Moreover, each entity illustrated in Figure 11 consists of variables/features which are expected to have values. For instance, considering the Symptom entity, it may have variables/features like Cough, Fever, Chest Pain and others. Each of those variables are expected to take values from a particular data type. Hence we further illustrated the class of data typing each of the entity may draw denotes the values of its variables. Potential data types as captured in Figure 11 are numeric, nominal, ordinals, datetime, and Boolean (which forms the largest representation for most values of variables in the representation).

\subsection{The CBR model}

All previous stages of the proposed CBR-based framework may be classified as data/input pre-processing and formalization operations. However, the main reasoning task is embodied in the CBR engine to be described in this 
section. Meanwhile, we shall first present a brief description of some status or clinical types of COVID-19 based on clinical presentation [50]:

Mild case: Upper respiratory symptoms such as pharyngeal congestion, sore throat, and fever for a short duration or asymptomatic infection; Positive RT-PCR test for SARS-CoV-2; no abnormal radiographic and septic presentation.

Moderate case: Mild pneumonia; symptoms such as fever, cough, fatigue, headache, and myalgia; and absence of complications and manifestations related to severe conditions.

Severe case: A case presenting with mild or moderate clinical features described above; rapid breath ( $\geq 70$ breaths per min for infants aged $<1$ year; $\geq 50$ breaths per min for children aged $>1$ year); hypoxia; lack of consciousness, depression, coma, convulsions; dehydration, difficulty feeding, gastrointestinal dysfunction; myocardial injury; elevated liver enzymes; coagulation dysfunction, rhabdomyolysis, and any other manifestations suggesting injuries to vital organs.

Critical illness case: Respiratory failure with need for mechanical ventilation, persistent hypoxia that cannot be alleviated by inhalation through nasal catheters or masks; septic shock; organ failure that needs monitoring in the ICU, and acute respiratory distress syndrome (ARDS). Cases presenting with ARDS may show:

i. Mild ARDS: $200 \mathrm{mmHg}<\mathrm{PaO} 2 / \mathrm{FiO} 2 \leq 300 \mathrm{mmHg}$. In not-ventilated patients or in those managed through non-invasive ventilation (NIV) by using positive end-expiratory pressure (PEEP) or a continuous positive airway pressure $(\mathrm{CPAP}) \geq 5 \mathrm{cmH} 2 \mathrm{O}$.

ii. $\quad$ Moderate ARDS: $100 \mathrm{mmHg}<\mathrm{PaO} 2 / \mathrm{FiO} 2 \leq 200 \mathrm{mmHg}$.

iii. Severe ARDS: $\mathrm{PaO} 2 / \mathrm{FiO} 2 \leq 100 \mathrm{mmHg}$.

These clinical types of COVID-19 have been described to allow for their use in the CBR engine which will be described below.

The CBR method is a reasoning paradigm that depends on a knowledge base of archived cases that have been proven and tested with valid solutions for handling new cases/problems which may share similar features with those archived. As earlier stated, this study builds on this paradigm to carry out the detection and diagnoses of COVID-19 in patients manifesting symptoms of the disease and those presenting with asymptomatic cases. Figure 12 illustrates our concept of the CBR engine embedded in Figure 8. The major components of the model are similar to the conventional CBR model which usually consists of the RETRIEVE, RESUSE, REVISE, and RETAIN steps (4Rs). In addition, the model shows the knowledge base of archived cases which allow for carrying out computational reasoning on the new case presented. The distinctiveness of our proposed CBR model lies in its ability to model its cases using ontology formalism and as well to measure similarity of cases using features listed in Table 2 and two other important factors: time (temporal) and spatial (location). We shall detail the operations in each level of the 4Rs in the following discussion. 


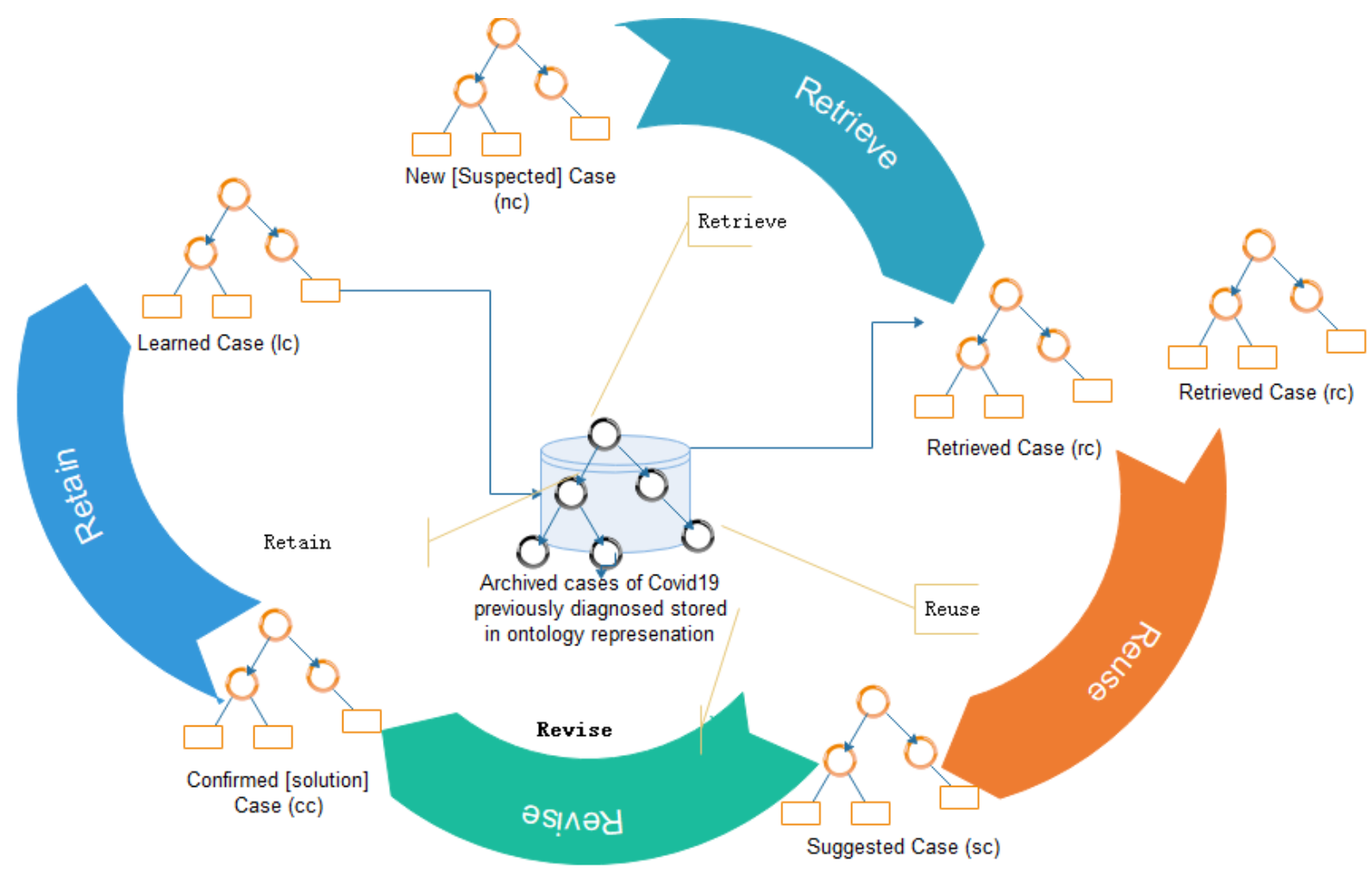

Figure 12: A model of the proposed case-based reasoning (CBR) used in the NLP-Ontology oriented Spatial temporal framework for detecting COVID-19

\section{A. Retrieve}

Based on the general concept of the CBR paradigm, the RETRIEVE procedure/algorithm simply uses some efficient distance or similarity computation models like the Euclidean distance, Cosine Similarity [51], and Manhattan distance. Our approach for the procedure of the RETRIEVE algorithm is described as follows: Consider new case $n c$ and an archive of stored cases in the CBR knowledge base $S C=\left\{s c_{1}, s c_{2}, s c_{3} \ldots . . s c_{n}\right\}$ such that the CBR model RETRIEVE the most similar $s c_{i}$ or some $s c_{i}$ from SC. However, the process of retrieval of some $s c_{i}$ depends on Equation 1. The smaller the value of $\operatorname{Sim}\left(n c, s c_{i}\right)$ the more acceptable the case $s c_{i}$ becomes for adoption for REUSE. Here is a summary of procedures in the RETRIEVE step:

i. Query generator and parser are used to construct a query that will fetch all similar cases from the case archive SC. The query(ies) is generated based on the extracted features in the previous stage of the framework described in Figure 8.

ii. Semantic Query Web Rule Language (SQWRL) (details later) is employed for modeling the constructed query in the preceding step.

iii. Output resulting from the SQWRL query is sorted in the order of the most similar to the least similar cases. Cases are assumed to be similar if their measure of look alikeness (based on the corresponding features) is non-negligible. The smaller the value of the similarity, the higher the likelihood of the new case $(n c)$ to share close similarity with a $s c_{i}$ or some $s c_{i}$, while the bigger the value of the similarity/distance metric, the lower its tendency to match up with $n c$.

iv. Hence, our problem can therefore be modeled as a classification problem whereby some $s c_{i} \in \mathrm{SC}$ are classified to share some similarity with $n c$ while another class of some $s c_{i} \in \mathrm{SC}$ are categorized among dissimilar cases. Execution of clinical similarity of cases is done by steadying the computation within the range of $[0,1]$ using the following equations:

- Euclidean distance: Describes the length between two points and is the most used distance/similarity metric with most appropriate for cases with continuous or dense data. Equation 1 models Euclidean distance. 


$$
E D=\sqrt{\sum_{k}^{n} w_{k} \cdot\left(f_{a k}-f_{b k}\right)^{2}}
$$

- Cosine Similarity: This similarity metric measures the dot product of the two features compared. Based on the cosine computation which yields 1 for $0^{0}$ and less than 1 for other degrees, it implies that a cosine similarity of 1 signals that features A and B are similar cases while a cosine value of 1 indicates non-similarity. Equation 2 models Cosine similarity which has strong application in data with sparse vectors. In addition, the Cosine similarity (CS) is able to perform that Euclidean distance (ED) in cases where ED sees two cases to be distantly similar; CS might observe a closer similarity among the two cases based on their oriented closeness.

$\operatorname{CSim}(x, y)=\frac{x \cdot y}{\|\mathbf{x}|||| \mathbf{y}\|}$

where $\|\boldsymbol{x}\|$ and $\|\boldsymbol{y}\|$ are the Euclidean norm of vector $\mathrm{x}=\left(\mathrm{x}_{1}, \mathrm{x}_{2}, \ldots, \mathrm{x}_{\mathrm{p}}\right)$ and Euclidean norm of vector $\mathrm{y}=\left(\mathrm{y}_{1}, \mathrm{y}_{2}, \ldots, \mathrm{y}_{\mathrm{p}}\right)$, respectively, and vector $\mathrm{x}$ defined as $\sqrt{x_{2}^{1}}+x_{2}^{2}+\cdots+x_{p}^{2}$.

- Manhattan distance: Another similarity or distance metric, also known as Manhattan length measures distance between points along an axis at a right angle. Equation 3 models Manhattan distance.

$$
M D=\left|x_{a k}-x_{b k}\right|+\left|y_{a k}-y_{b k}\right|
$$

- Other similarity measures are the Jaccard similarity (use for sets) and Minkowski distance equations.

Now, because our cases in the proposed framework were modeled/formalised in ontology represenation, there was a need to be able to carry out quantitative measures of similarity between features of cases, hence the need to use ontology-based semantic similarity between terms. There are six (6) major techniques for computing such similarities of features in ontology: ontology hierarchy approach, information content, semantic distance, approach based on properties of features, approach using ontology hierarchy, and hybrid methods [52]. Therefore, to compare two cases, we make the following assumptions:

i. Two cases are similar if their ontologies demonstrate similarities in both feature values and structure (of their ontological representations).

ii. That an arbitrary weight $\boldsymbol{w}_{i}$ value is assigned to each property (object and data properties) in their (cases to compare) ontologies (which must sum to 1 for each ontology representing a case). For example, all properties/relations/links (denoting object properties) as shown in Figure 11 from the Case node to the other nodes (Diagnoses Case, Symptoms, Epidemiology, Radiology/Laboratory, Clinical Diagnoses, and Treatment) each will be assigned a weighted value. Similarly, links (data properties) from the second level (Diagnoses Case, Symptoms, Epidemiology, Radiology/Laboratory, Clinical Diagnoses, and Treatment) to the lower level (values) also have weight values summing up to 1 . For instance, the presentsSymptom (object property) may have weight 0.3 , hasEpidemiology (object property) may have such that each symptom weight 0.2 , and so on until all second level nodes have weights summing up to 1 . However, a case may present $\boldsymbol{n}$ features associated with the presentsSymptom link, i.e a case with cough, fever, Anosmia and ARDs (which are all symptoms). So, we also assign weights $f \boldsymbol{w}_{i}$ to each of the known symptoms such that only the weight of the present symptoms in an arbitrary case $[c]$ are summed up all $f \boldsymbol{w}_{i}$ for such case and multiply it with its second level (Symptom node) weight $\boldsymbol{w}_{i}$. Hence, each mention/use of the notation $\boldsymbol{W}_{i}$ in our Equation 6 will denote summation of all $\left(\boldsymbol{w}_{i} . f \boldsymbol{w}_{i}\right)$

Note: all our objects (such as presentsSymptom and hasEpidemiology) and data type properties are detailed and discussed in Section 4.3

iii. We modeled all features in Table 2 according to their expected inputs. For example, some features are either present or absent and those features with values bounded between a range (e.g. Cough: Y/N, Oxygen 
therapy: Y/N, Heart rate: $>=88.63$, Temperature $\left.\left({ }^{\circ} \mathrm{C}\right):>=37.86\right)$ are modeled with 1 or 0 and values from $[0.0,1.0]$ respectively. Hence, each $f \boldsymbol{w}_{i}$ described in (ii) above affected by their impact is denoted by $\boldsymbol{p}$. Hence, the true value of $f w_{i}$ is :

$$
f w_{i}=. f w_{i} \cdot p
$$

Now that we have established our distance/similarity functions and basoc assumptions for case retrieval, here is the formula for computing similar cases in the archived compared to new case $(n c)$. This study adopts the approach based on properties and features described in [52]. The adapted similarity measure is that of Tversky (1977) as shown in Equation 4:

$$
\operatorname{Sim}\left(n c \mid s c_{i}\right)=\frac{\left|D_{1}+D_{2}\right|}{\left|D_{1} \cap D_{2}\right|+\mu\left|D_{1} / D_{2}\right|+(\mu-1)\left|D_{1} / D_{2}\right|}
$$

The model in equation (5) assumes that $n c$ and $s c_{i}$ are cases whose features are collected in $\mathrm{D}_{1}$ and $\mathrm{D}_{2}$ respectively. Therefore, the similarity between $n c$ and $s c_{i}$ is computed using three components of equation (5): distinct features of $n c$ to $s c_{i}$, distinct features of $s c_{i}$ to $n c$, and common features of $n c$ and $s c_{i}$, for $0<=\mu<=1$, a function that defines the relative importance of the non-common features. D1 and D2 represent the target and the base respectively while $\|$ stands for the cardinality of set. Although we approve of the similarity model in Eq. 5, we however saw its limitation which is based omission of the effect of weight on selected features. Therefore, we modified Eq. 5 so that we do not use the elements of the set alone, but the weight-value of the elements in each $\mathrm{D}_{\mathrm{i}}$ which is computed by Eq. 6 . Hence, our modification to Eq. 5 is shown in Eq. 7, afterward, the most similar $s c_{i}$ is RETRIEVE and forwarded to REUSE after applying Eq. 8 .

Where $\mathrm{D}_{1}$ or $\mathrm{D}_{2}$ computed using Eq. 6:

$$
\begin{gathered}
D=\sum_{i=0}^{n} \boldsymbol{w i} \cdot \boldsymbol{f} \boldsymbol{w i} \\
\operatorname{Sim}\left(n c \mid s c_{i}\right)=\frac{D_{1}+D_{2}}{D_{2} \cap D_{1}+\mu D_{1} / D_{2}+(\mu-1) D_{2} / D_{1}}
\end{gathered}
$$

Furthermore, since $\operatorname{Sim}\left(n c \mid s c_{i}\right)$ represents our similarity between new case $(\boldsymbol{n c})$ and an arbitrary case $s c_{i}$ in the archived, we can compute the similarity score $(S S)$ using Eq. 8.

$$
\text { Similarity Score }(S S)=1-\operatorname{Sim}\left(n c \mid s c_{i}\right)
$$

Hence, cases with with $\boldsymbol{S S}$ close to 1 are similar to $\boldsymbol{n c}$ and as result such cases are retrieved. However, if no case is retrieved by Eqs. 7 and 8, we then conclude that $\boldsymbol{n c}$ might not have any similar case.

We further compute $S S$ for positive and negative cases and apply Equations 9 and 10 to determine the following: When $S S_{\text {covid19+ }}>S S_{\text {covid19- }}$ the case is classified as positive case of COVID-19, while if $S S_{\text {covid19+ }}<$ $S S_{\text {covid19- }}$ the case is concluded to be negative. However, an evaluation of $S S_{\text {covid19+ }}=S S_{\text {covid19- }}$ indicates an inclonlusive diagnoses, therefore necessitating more similar case(s) be retrieved.

$$
\begin{aligned}
& S S_{\text {covid19+ }}=\sum_{k=0}^{n}\left(S S^{+}\right) \\
& S S_{\text {covid19- }}=\sum_{k=0}^{m}\left(S S^{-}\right)
\end{aligned}
$$




\section{B. Reuse}

The REUSE procedure allows the system to modify the RETRIEVE cases $\boldsymbol{s} \boldsymbol{c}_{\boldsymbol{i}}$ in such a manner that we have only one similar case. The similar case is constructed to maintain a similar ontology structure with the $n \boldsymbol{c}$ case. This is achieved by rebuilding an anonymous case $(\boldsymbol{a c})$ by extracting all similar features of the presented cases in $s c_{i}$ until $\boldsymbol{a c}$ assumes the form of $\boldsymbol{n c}$. As such the modified $\boldsymbol{a c}$ is presented as a temporary solution to $\boldsymbol{n c}$. The approach proposed here is different from methods used by $\mathrm{Gu}$, et al. [53], which relied on clinical protocols guidelines and medical experts respectively. The $\boldsymbol{a c}$ case is therefore considered a solved case which will be passed on to the REVISE step for processing.

\section{Revise}

The evaluation of $\boldsymbol{a c}$ case at this stage is achieved by ensuring that the summation of case features of the proposed solution case is not greater than 1 . If they evaluate to more than 1, some non-essential features are dropped and the weights of the features are recomputed until an appropriate value is obtained. The revised and evaluated case now becomes a candidate case for use, and it is called the repaired case $(\boldsymbol{r c})$. Furthermore, $\boldsymbol{r c}$ is then used to solve the new problem $\boldsymbol{n c}$ presented to the system. The solution to $\boldsymbol{n c}$ is passed to the RETAIN.

\section{Retain}

Finally, the RETAIN procedure simply stores the solution to $\boldsymbol{n c}$ as a case that has been learned and is fit to be stored/added to the knowledge base of CBR model for future use.

\subsection{Algorithm for Case Retrieval}

Algorithm 1 details the complete procedure outlined in proceeding subsections, and describes how a new case of COVID-19 is classified as a positive or negative case using the CBR method. The input to the algorithm is an HER of the new case and the out is Diagnoses Case (Suspected, Confirm, Presumed status).

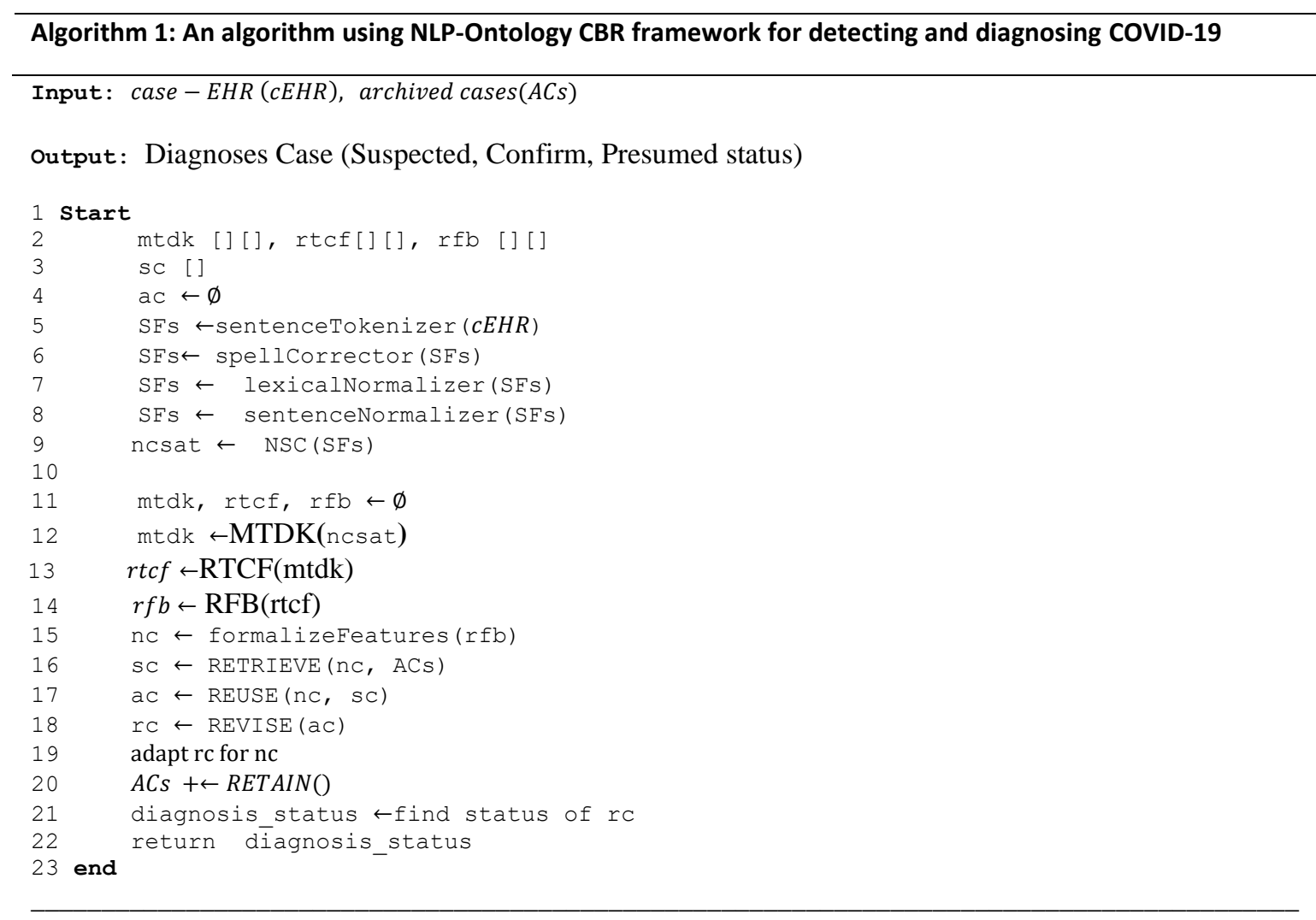

\subsection{Experimentation}

In this section the clinical data and experimentation environment used in this study are described. In addition, we develop the domain ontology (for Covi19 and other related Covs-based disease) and also the case-based ontology for new cases. Finally, we demonstrate the implementation of the framework as shown in Figure 8. 


\subsection{Clinical Data}

The COVID-19 pandemic is currently a global emergency with limited access to health facilities and even computerized patient records which could have allowed access datasets for computational research. Although there are statistical-based datasets accessible in the forms of structured, semi-structured, and unstructured (e.g. WHO, Johns Hopkins University, mainstream news media, and even social media), however, such datasets are still unfit for tasks like the one in this study. After a thorough search for publicly available patient HER-based benchmarked datasets of COVID-19 with none accessible, we decided to adopt the approach of curating new datasets of COVID19 from some available data on standard domains.

The data curated was obtained from the Italian Society of Medical and Interventional Radiology (SIRM). SIRM is a scientific association which includes the majority of Italian radiologists, and is targeted to encourage the progression of diagnostic imaging by promoting studies and research. The data source (https://www.sirm.org/en/italian-societyof-medical-and-interventional-radiology/) listed English-like records (itemizing age, symptoms and signs manifested, and other laboratory details) and CT scans for each of the sixty-eight (68) COVID-19 patients. We anonymized and cleaned the datasets where necessary and extracted the necessary information, storing them in a format appropriate for this study. Figure 13 shows snapshots of a randomly selected case.
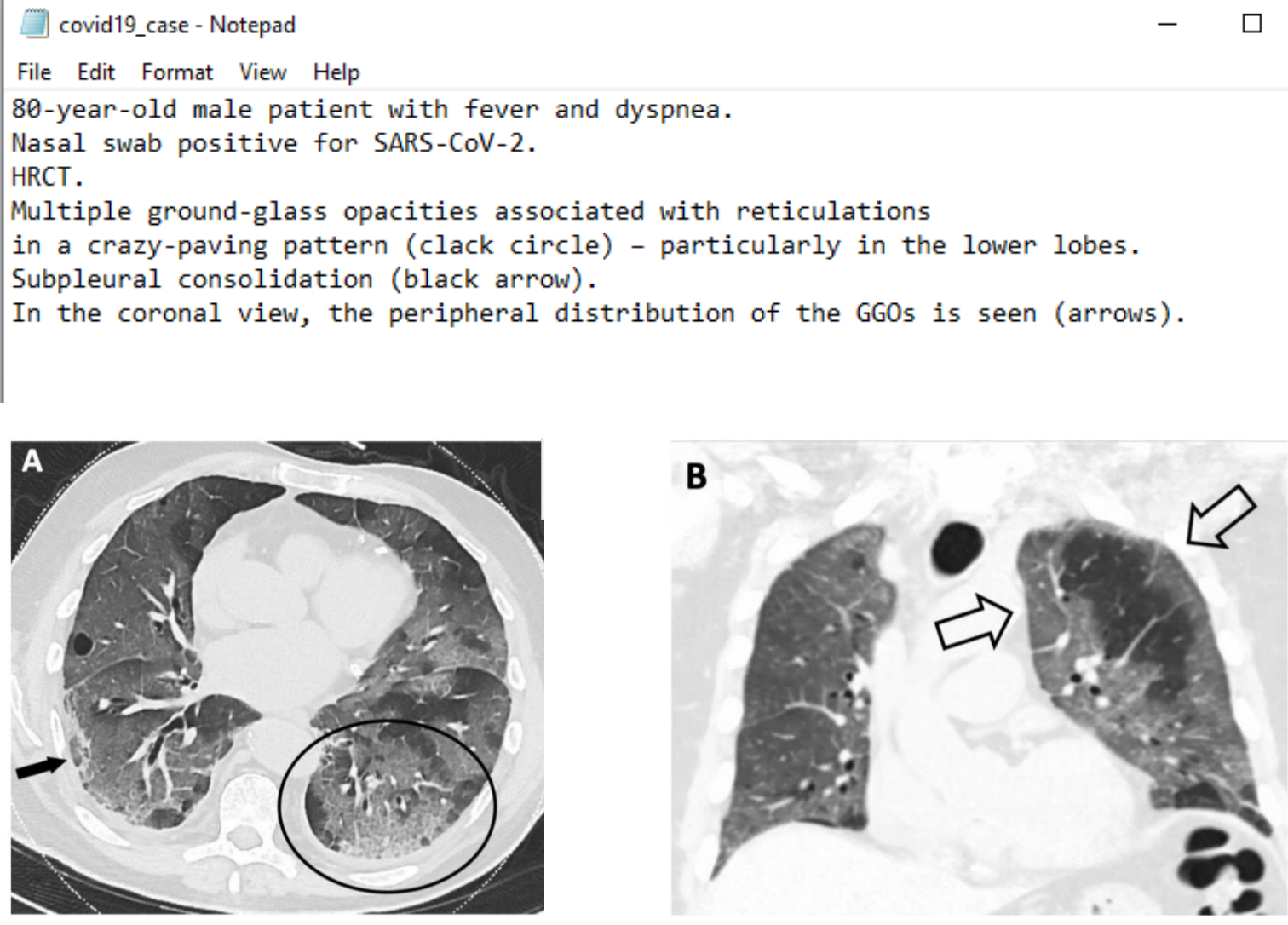

Figure 13: Dataset of a sample case of COVID-19 showing some English-like statements extracted and samples of CT scans and X-ray performed on the patient.

The data need of this study is EHR-based datasets in natural language (NL) format. Hence, we focused on processing the English-like statements extracted for each patient leaving the image-based for future study using the approach of deep learning for classification of COVID-19 cases.

\subsection{Computational Environment Setup}

The implementation was on a personal computer with CPU of Intel (R) Core i5-4210U CPU $1.70 \mathrm{GHz}, 2.40 \mathrm{GHz}$; RAM of 8 Gbytes; Windows 10 OS. Furthermore, we deployed Anaconda shipped with Python 3.7.3, SPIDER 3.3.6, 
and also installed NetBeans IDE version 8.1. The Python platform allows for the implementation of the NLP feature extraction pipeline shown in Figure 9 while the NetBeans IDE provides support for implementing the feature to ontology representation and also the CBR-engine. Modeling of ontologies in this study was achieved using Protégé (Protégé).

\subsection{Domain Ontology Modeling}

Ontologies are formalism for specification of concepts or abstract description of a system in a domain-specific knowledge composition. Ontologies as formalism stems from description logic (DL) and with support for reasoning it has received OR has caused it to receive more and more attention in computational biology and bioinformatics. There are different ontology languages like RDF/RDFS, DALM+OIL, and OWL. OWL is a DL-based ontology language with high expressivity and has three variants: OWL-DL, OWL-full and OWL-lite. This study models ontologies using OWL2 [54, 55], which is an improved version of OWL (sometimes known as OWL1). We have modeled three different ontologies: the first represents domain knowledge, the second is a formalism of the archived cases, and the third ontology formalizes new cases.
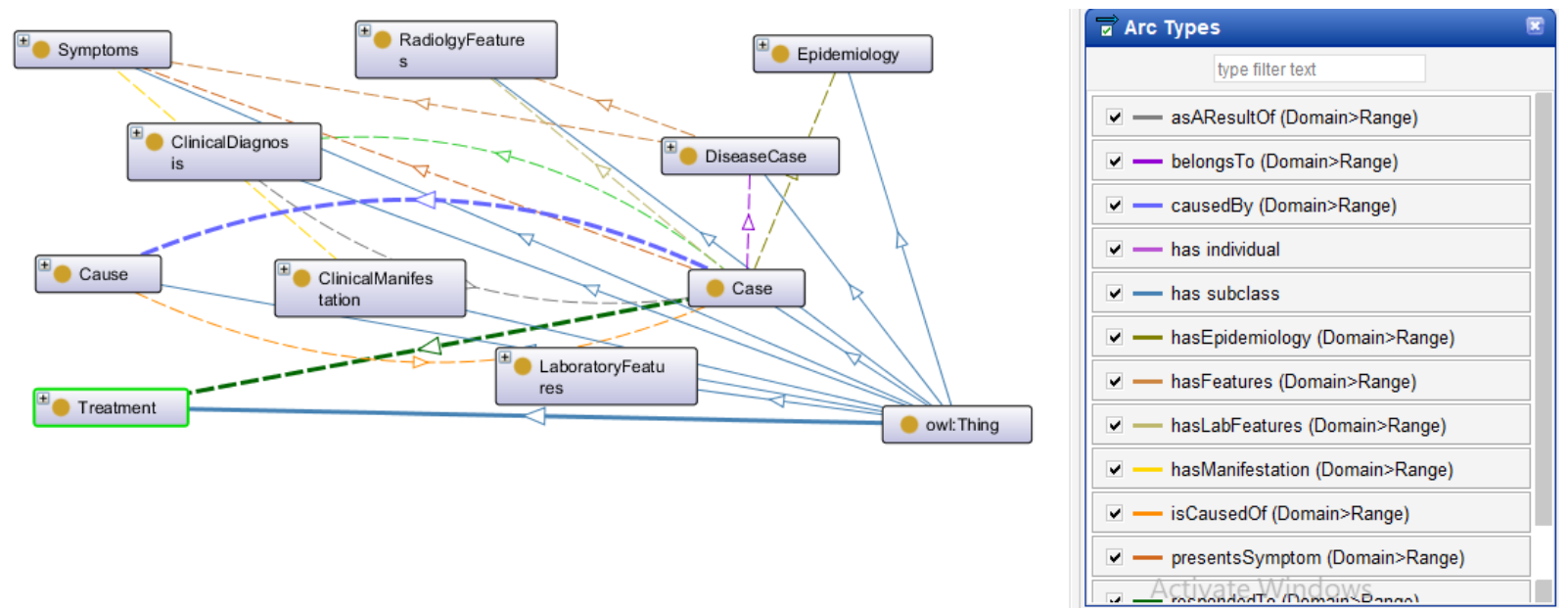

Figure 14: Ontology representation domain-based mapped tokens (features) of a new case of coronavirus (COVID19): New Case (nc)

In Figure 14, we show a visualization of the ontology representing a new case (nc). The ontology captures concepts/classes like Symptoms, ClinicalDiagnosis, ClinicalManifestation, Epidemiology, RadiologyFeatures, LaboratoryFeatures Case (to denote a new case), DiseaseCase, Cause (to capture the likely causes of the disease in a case), and Treatment (which represents treatments administered to a case). Each of these concepts is related/linked to another concept by a property (object property) with almost all concepts linked to Case. To the right is a list of the object properties. For example the line connecting Case to Symptoms is the object property presentSymptom. The Case is the domain while Symptoms is the range for the object property presentSymptom. Some concepts have the + symbol at the top-leftmost corner of their bounding boxes. This is an indication that there are other subclasses in that concept/class which can be revealed by clicking on the + symbol.

Case formalization is therefore made possible through the Case-Based ontology file shown in Figure 14. While that illustrates a case of COVID-19, we made a further effort to use an ontology approach to model the archive of stored cases in the CBR engine. To archive this, we represented the structure and the semantic of the information content of such archive using the ontology visualized in Figure 15. As mentioned earlier, the ontology file was modeled and visualized in Protégé (Protégé). The ontology consisted of 459 axioms, 225 logical axioms, 213 declaration axioms, 196 Class, 11 object property, 8 data type property, 181 subclasses, and 15 instances (with the exception of cases of COVID-19 which forms the archive of cases in the ontology). Figure 15 captures the is-a relationship existing among classes and Figure 16 outlines all the classes (and their hidden subclasses), instances (individuals) of the declared classes, object and data type properties. 


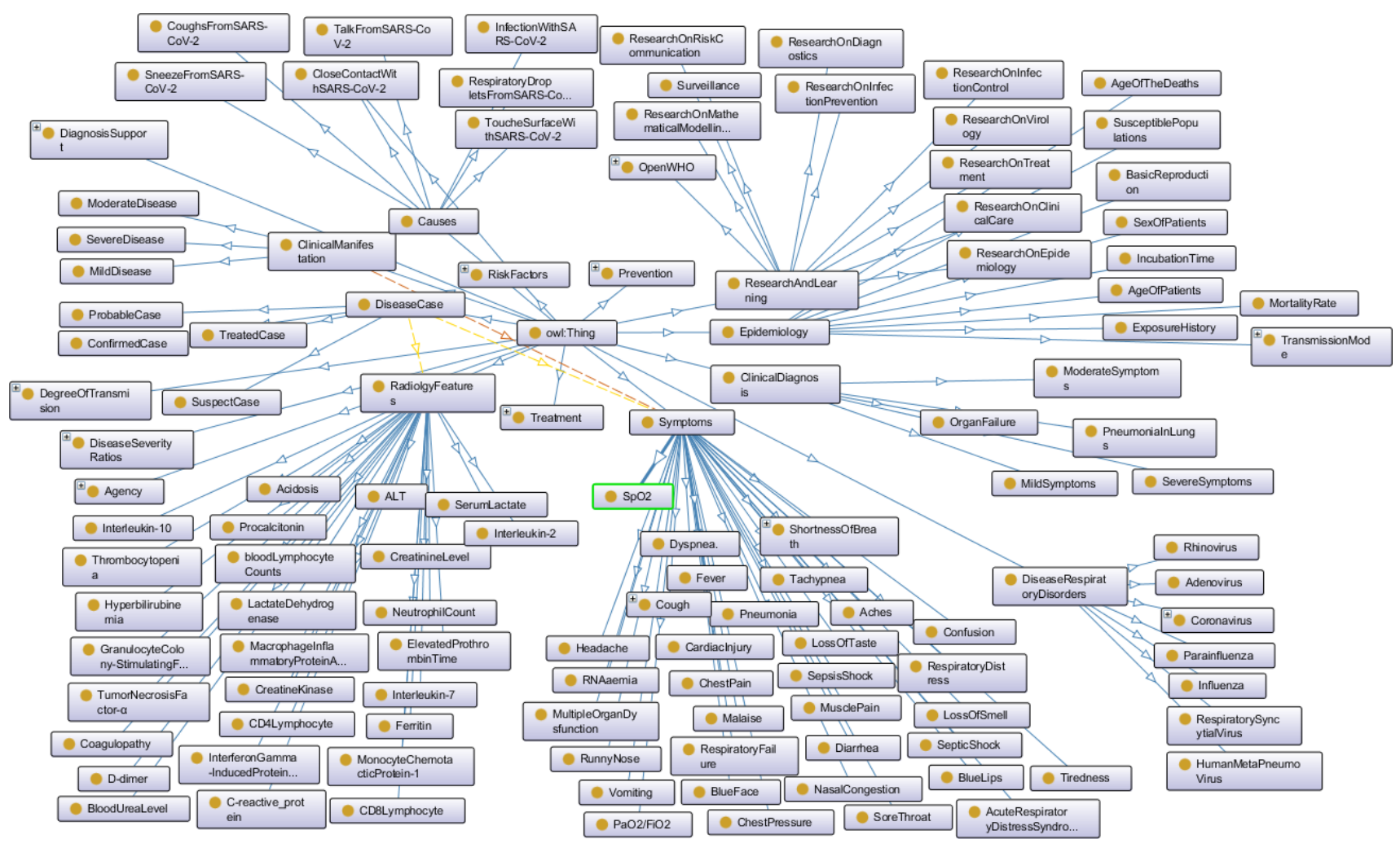

Figure 15: A visualization of ontology representation of relations of concepts (TBox) in a domain-based knowledge repository of COVID-19 using the is-a relationship

Class hierarchy:
Causes
ClinicalDiagnosis
ClinicalManifestation
DegreeOfTransmision
DiagnosisSupport
DiseaseCase
DiseaseRespiratoryDisorders
DiseaseSeverityRatios
Epidemiology
Prevention
RadiolgyFeatures
ResearchAndLearning
RiskFactors
Symptoms
Treatment

ए曰日口

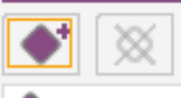

AerosolDroplets

AGenerallntroductionToEmergingRespiratoryViruses

ClinicalCareForSevereAcuteRespiratorylnfections

Covid19

Covid19-OPG

Covid19-PP

DifficultyBreathing

DryCough

EPROTECT

HumanToHumanFomites

IPCNC-Covid19

MildCough

NosocomialTransmission

PhysicalContact

ZoonoticTransmission 


\section{Data property hierarchy: owl:topD}
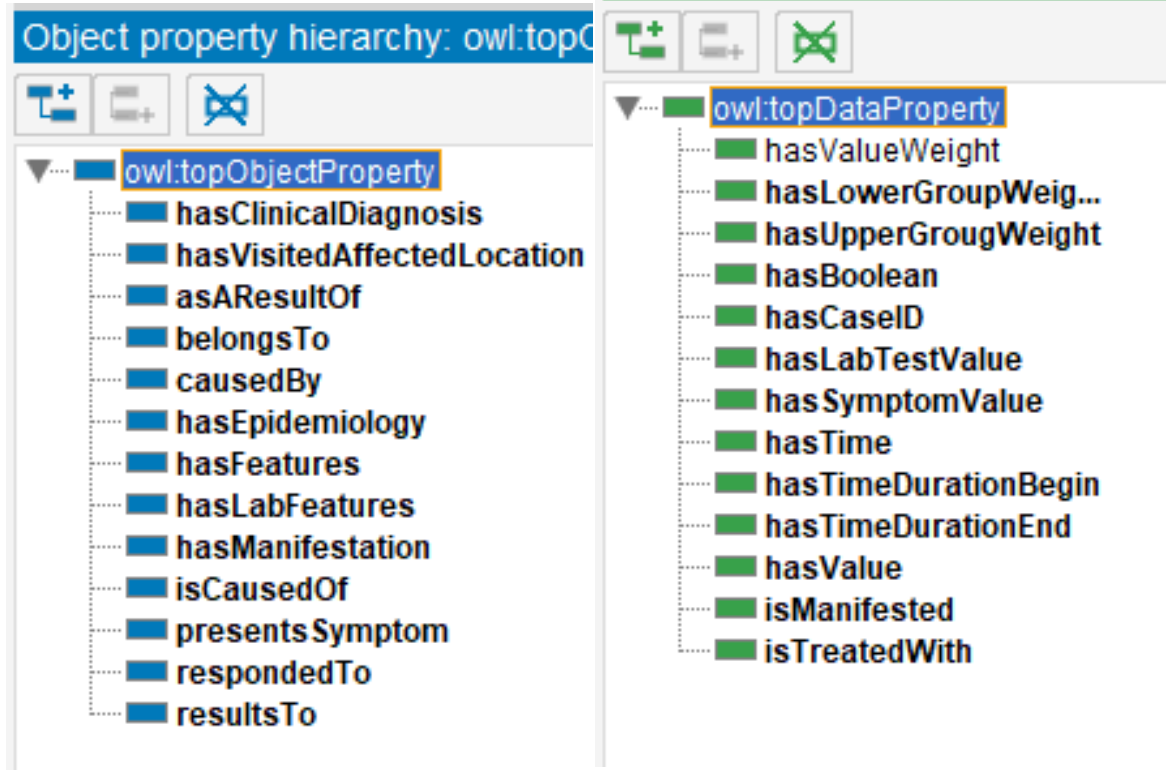

Figure 16: A listing of concepts (classes), individuals (instances of classes), object properties and data type properties modeled in a domain-based knowledge repository of COVID-19

Now that we have formalism for archiving all cases in the proposed framework and also a formalism for modeling new cases extracted from electronic records, we shall consider how the proposed approach will implement its query for similar cases as modeled using mathematical models in item A of subsection 3.4. To archive an optimized and effective query of cases from the archive, we decided to construct our query from the mathematical models presented earlier using Semantic Query-Enhanced Web Rule Language (SQWRL), pronounced squirrel. SQWRL is a query language primitive to OWL and also an SWRL-based with syntax of SQL-like and having operators for extracting information from OWL ontologies [56, 57]. We chose SQWRL over SPARQL because of its suitability for use in OWL ontologies since it does not require serializing our OWL ontologies in RDF/RDFS, an operation which often causes a knowledge-base (ontology) to lose some semantics and expressivity as a result of serialization. Moreover, the rule-form of SQWRL and its compatibility with the rule language SWRL allows for improving our framework to use inference engine, thereby improving the knowledge-base through inference. Protégé also provides a tab for executing our SQWRL queries against the ontology through the SQWRLTab plugging. We may take advantage of this tab to text our generated queries, although the framework proposed in this study has a mechanism for doing the query execution automatically through OWLAPI.

Now for instance, given a new case (nc) presenting with the following features according to their category, we might be interested in translating our mathematical model into an SQWRL query such that similar cases are retrieved: Symptoms (Cough, Temperature, Nausea and vomiting, Shortness of breath, Contact with case(s)); Laboratory Features(Neutrophil, Lymphocyte, Active partial thrombin time);

The following conditions can be assumed from our case: retrieve all cases according to their value of similarity (in descending order), which have values for all or some of the features: Symptom (Cough, Temperature, Nausea and vomiting, Shortness of breath, Contact with case(s)); Laboratory Features (Neutrophil, Lymphocyte, Active partial thrombin time). In Figure 17, we present a sample SQWRL-query for extracting similar cases compared to the new case example described here. 
Case $(? \mathrm{c})^{\wedge}$ hasCaselD(?c, ?cid)

${ }^{\wedge}$ Cough(?sc) ${ }^{\wedge}$ hasLowerGroupWeight(?sc, ?scwi) ${ }^{\wedge}$ presentsSymptom(?c, ?sc) ${ }^{\wedge}$ hasBoolean(?sc, ?cv1)

${ }^{\wedge}$ Temperature(?st) ^ hasLowerGroupWeight(?st, ?stwi)^ presentsSymptom(?c, ?st) ^ hasSymptomValue(?st, ?cv2)

${ }^{\wedge}$ Nausea(?sn) ^ hasLowerGroupWeight(?sn, ?snwi)^ presentsSymptom(?c, ?sn) ^ hasBoolean(?sn, ?cv3)

${ }^{\wedge}$ Vomiting $(? \mathrm{sv})^{\wedge}$ hasLowerGroupWeight(?sv, ?swwi)^ presentsSymptom(?cid, ?sv) ${ }^{\wedge}$ hasBoolean(?sv, ?cv4)

$\wedge$ ShortnessOfBreath(?ssb)^ hasLowerGroupWeight(?ss, ?sswi)^ presentsSymptom(?c, ?ssb) ${ }^{\wedge}$ hasBoolean(?ssb, ?cv5)

${ }^{\wedge}$ Covid19AffectedLocation(?loc) ${ }^{\wedge}$ hasVisitedAffectedLocation(?c, ?loc)

${ }^{\wedge}$ Neutrophil(?In) ${ }^{\wedge}$ hasLowerGroupWeight(? In, ? Inwi) ^hasLabFeatures(?c, ?ln $)^{\wedge}$ hasLabTestValue(?In, ?cv6)

${ }^{\wedge}$ Lymphocyte(?II) ^ hasLowerGroupWeight(?I, ? ?lwi) ^ hasLabFeatures(?c, ?II)^ hasLabTestValue(?I, ?cV7)

${ }^{\wedge}$ ActivePartialThrombinTime(?laptt) ${ }^{\wedge}$ hasLowerGroupWeight(?laptt, ?lapttwi) ${ }^{\wedge}$ hasLabFeatures(?c, ?laptt) ${ }^{\wedge}$ hasLabTestValue(?laptt, ?cv8)

${ }^{\wedge}$ hasTimeDurationBegin(?c, ?time1)

${ }^{\wedge}$ hasTimeDurationEnd(?c, ?time2)

${ }^{\wedge}$ hasClinicalDiagnosis(?c, ?status)

${ }^{\wedge}$ respondedTo(?c, ?tr)

^ swrlb:greaterThan(?cv2, 38)|

> sqwrl:select(?c, ?cid, ?loc, ?cv1, ?cv2, ?cv3, ?cv4, ?cv5, ?cv6, ?cv7, ?cv8, ?time1, ?time2, ?status, ?tr, ?scwi, ?stwi, ?snwi, ?swwi, ?sswi, ?Inwi, ?llwi, ?lapttwi)

${ }^{\wedge}$ sqwrl:orderBy(?cid) ${ }^{\wedge}$ sqwrl:count(?c)

Cancel Ok

Figure 17: A sample SQWRL query constructed to retrieve similar cases corresponding with the new case ( $n c$ ) accepted as input.

The sample query in Figure 17 was submitted to the Protégé application for execution and query of the underlying ontology through the SQWRLTab plugins. The syntax of the SQWRL query language aligns itself to the declared classes or entities, properties (both data type and object), and instances/individuals on the ontology. This is why you will observe that the predicates (unary and binary) names in the listed query in Figure 17 derive their values from the declared classes or entities, properties (both data type and object), and instances in the ontology. This positions the SQWRL query above the use of SPARQL. A detail explanation of the query given in the following lines:

The first line Case (?c) ^ hascaseID (?c, ?cid) extracts all cases and their case IDs from the CBR case archive and stores those two values in the (?c, ?cid variables. Furthermore, the second lines 2-5 of our query select instances of the following symptoms which were keywords/features extracted from the natural language input above: Cough, Temperature, Vomiting, and ShortnessofBreath, and their weight values. This is summarized in the following lines:

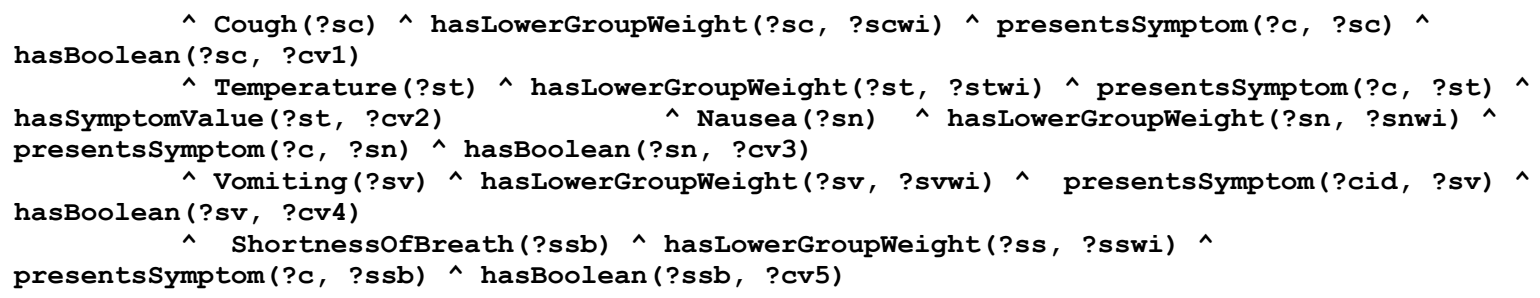

Also, our natural language based query has some laboratory features which we also extracted their values for each of the cases retrieved due to the query on line 1 . These laboratory features are queried as follows:

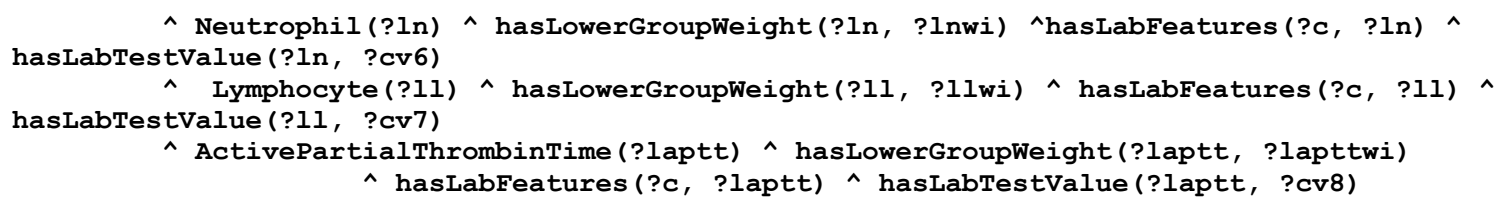


We are also interested in the probable points/places of contacts/visited by the cases that have been retrieved so far. Hence the line below:

$$
\text { ^ Covid19AffectedLocation(?loc) ^ hasVisitedAffectedLocation(?c, ?loc) }
$$

Now that all existing cases in the archive satisfying the above conditions have been retrieved, we further limit the cases to be extracted to the conditions below:

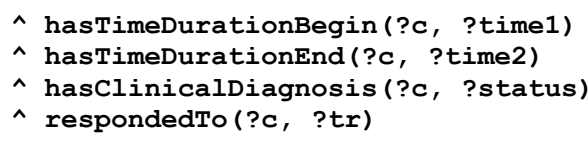

The first and second lines simply ensure the cases retrieved have the time/date when the case manifested and either died or recovered. Line three also allows each case to fetch the result of its clinical diagnosis (Positive or Negative diagnosis). Finally, the respondedTo(?c, ?tr) predicate fetches the treatment (if any) options recorded against each case.

Once all these cases are matched by the rule-like left-hand-side (LHS) of our query (a simulated of semantic web rule langue SWRL), the right-hand-side (RHS) uses the sqwrl:select predicate to fetch all cases (and their attributes/features) satisfied by LHS using the variables. Hence the lines below:

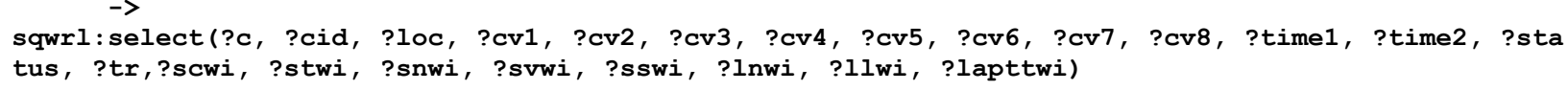

Finally, we are interested in counting the number of cases retrieved after ordering them according to their case IDs. The line of query below does this:

$$
\wedge \text { sqwrl:orderBy(?cid) ^ sqwrl:count(?c) }
$$

All cases retrieved by the sample query above must have its features represented in the ontology for the query to be able to match them. Case representation is covered in Section 3 of this paper, however, we have captured in Figure 18, a formalization of sample patient record shown in Figure 13. The case representation shown here is a Protégé interface format of the case, although the ontology notational is equally generated.

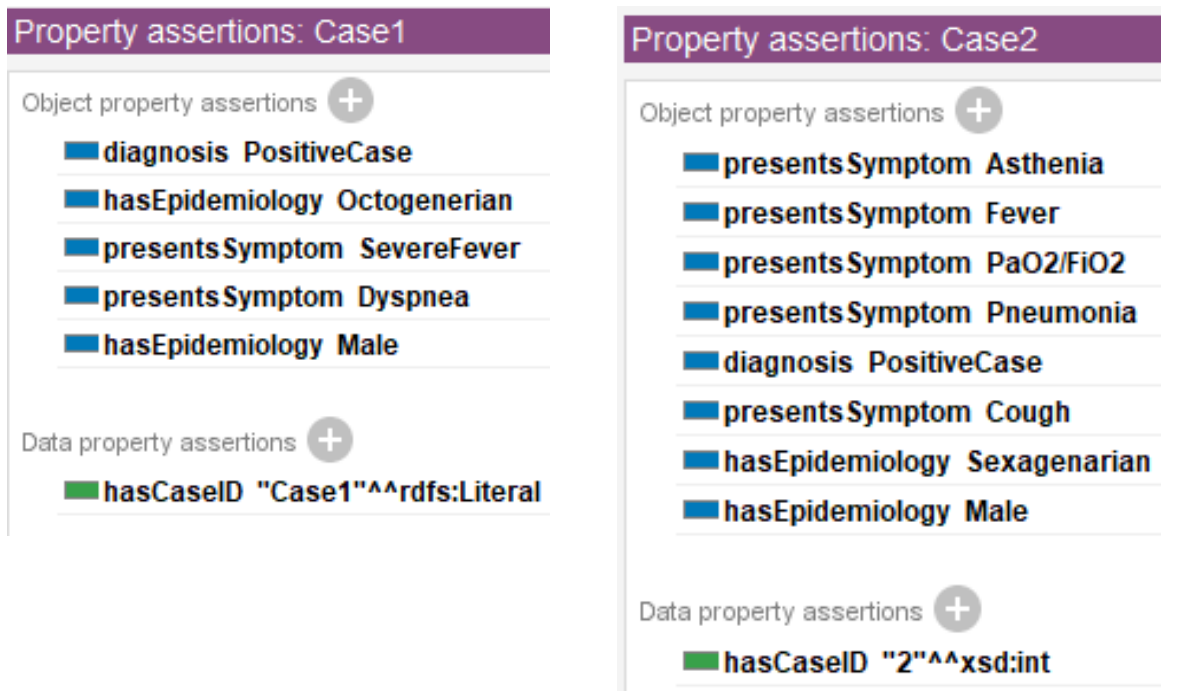

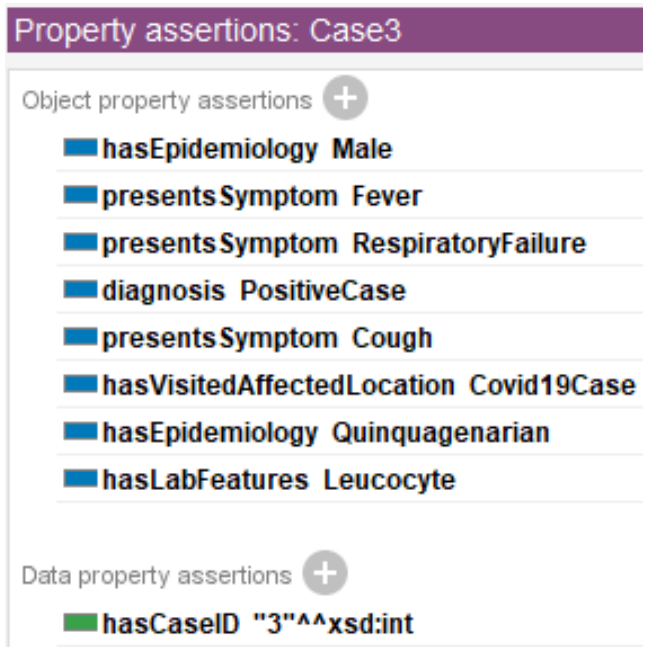

Figure 18: An illustration of case representation as shown in Protégé for cases a 1, 2, and 3 from the 68 cases extracted from the data source

\subsection{Implementation and Experiments}

The implementation of the CBR framework proposed in this study adopted JCOLIBRI [58]. Jcolibri is a library containing APIs for implementing a CBR framework and is written in Java. As a result, we employed the use of 
Java programming language to develop the CBR-engine (shown in the right box or component of Figure 19), Python programming to implement the natural language to Normalized Sentence Component (NL-NSC), and finally, a combined use of the two languages made the implementation of the feature extraction and formalization components of Figure 19 possible.

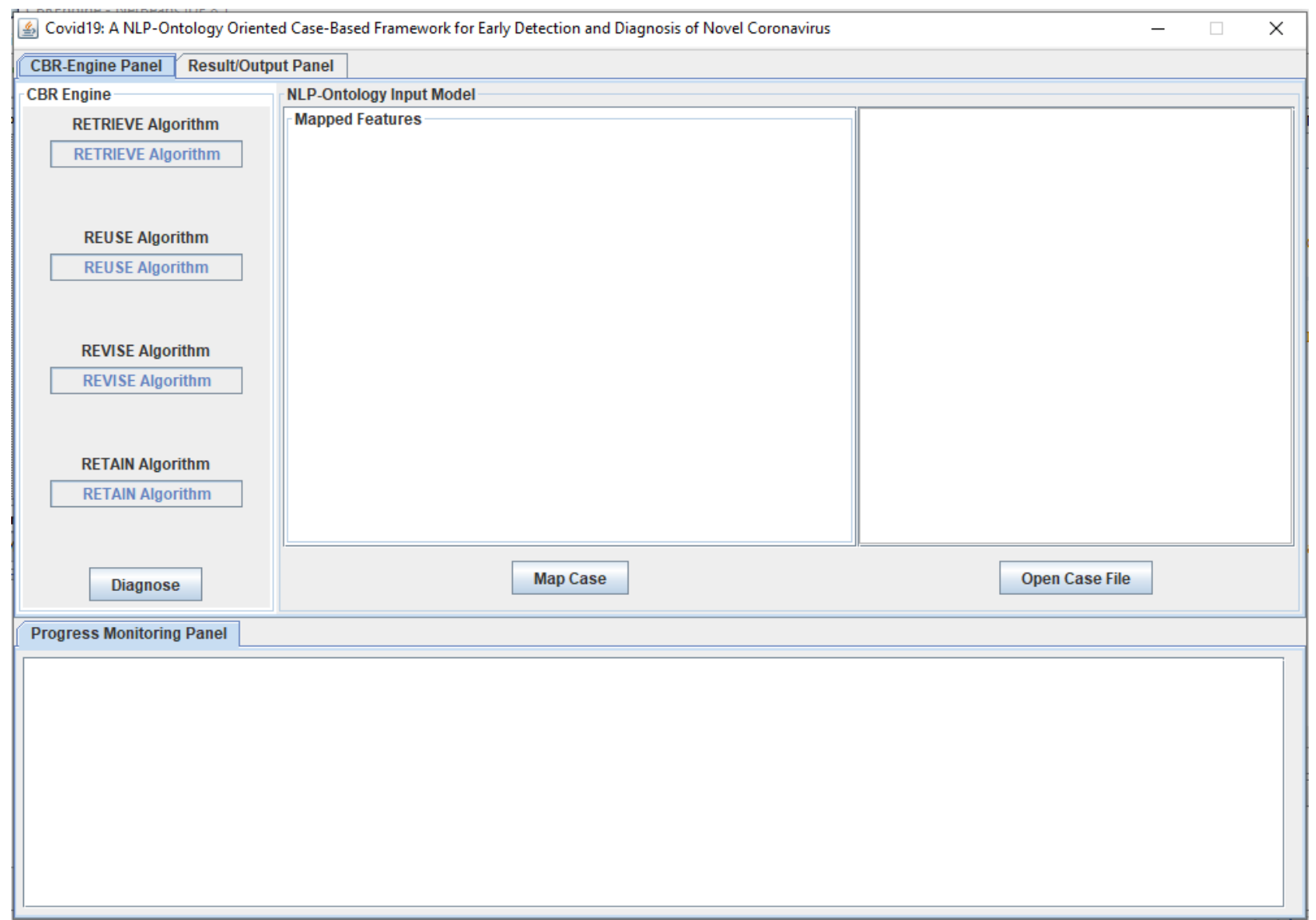

Figure 19: A graphical user interface (GUI) showing the major components of the proposed CBR-based framework for classifying cases of COVID-19 as either positive or negative case.

The complete implementation of the proposed framework is accessible through a graphical user interface (GUI) designed for this study and shown in Figure 18. The file loader and raw text extraction component of Figure 9 is implemented in the rightmost panel with a box and 'Open Case File' button in Figure 19. Furthermore, from Figure 16, the center panel containing a box and 'Map Case' button captures the implementation of the NL-NCS, feature extraction, and feature formalization components identifiable from Figure 11. To achieve this, standard Python libraries and NL-based libraries (like NLTK and Stanford CoreNLP) were richly employed to carry out the tasks of sentence disambiguation, spelling correction, lexical normalization, and normalization of sentences into their corresponding structures or components, and tokenization of sentences to enhance the process of feature mapping. However, the feature mapping and formalization of cases in ontology format was achieved using OWLAPI, Wordnet API, and Pellet API (an OWL-based knowledge reasoning plugin) which were implemented through a skillful use of Python and Java.

The result of the extracted and mapped features presented us with a challenge of accurately extracting values from the processed patient record. For example, we could have extracted features like 'Fever', 'Temperature' and so many other features which largely rely on syntax and semantic parsing of domain lexicon. But the challenge we were faced with was detecting the semantics/meaning and context of usage of the features from the patient records. To circumvent this, we took advantage of the named entity resolution technique we applied to the text. At a sentential-level, an attempt was made to search for values of features within the neighborhood of that feature. For instance, given the sentence: 
'The temperature of the patent was $38^{\circ}$ ',

a careful parsing of the sentence using NLP technique will reveal that the feature (temperature) has 38 degrees Celsius. But consider the sentence:

'80-year-old male patient with fever and dyspnea.'

There are two features in the sentence (fever and dyspnea) which do not have explicit declaration of values assigned to them. In cases like these, we developed a sentiment analysis component which enabled us to detect if such features were stated in the affirmative or negative form. The outcome of our sentiment analysis model outputs was: positive, negative and neutral. These outputs were used accordingly to formalize the feature and its value (true or false, as shown in Figure 16 and Table 2) in the ontology.

The leftmost panel of Figure 19 illustrates the implementation of the CBR-engine. This CBR-engine and the mathematical model presented in Section 3 were implemented with Java using the jcolibri API which models the Retrieve, Retain, Revise, and Reuse (4Rs) of CBR paradigm, allowing for users to adapt it to their frameworks. Meanwhile, we have also added a panel for monitoring the procedures for detection of status of any presented case of COVID-19; this monitoring begins with file loading component to the CBR-engine processes.

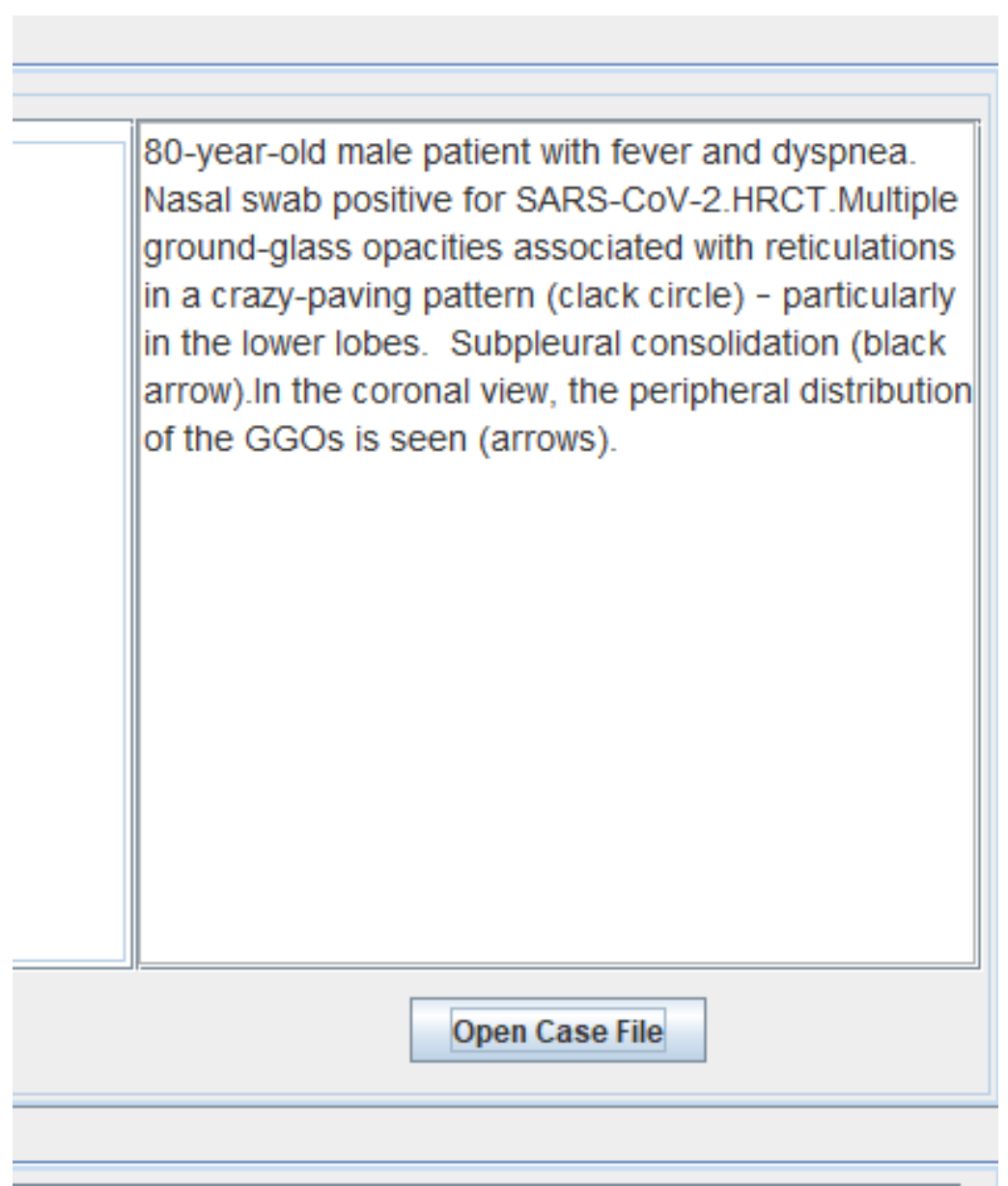

Figure 20: A demonstration of the File Loader component of the proposed framework 


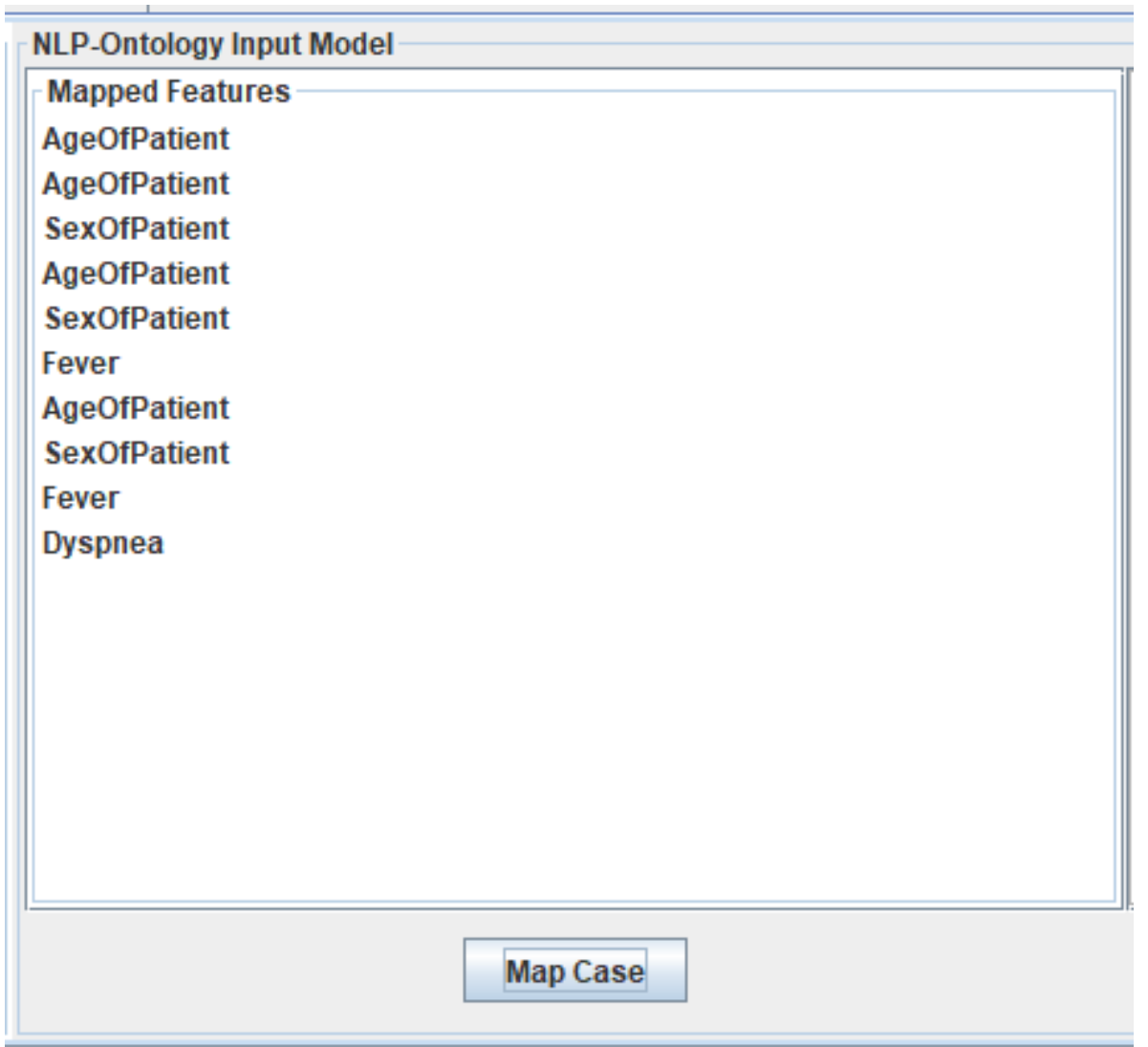

Figure 21: A demonstration of the Feature Mapping component of the proposed framework

Experimentation using the datasets discussed in Section 4.1 revealed that the implementation of the proposed CBR framework was successful. Figures 20 and 21 show a demonstration of the File Loader and Feature Mapping components of the CBR framework. Meanwhile, the process of formalizing feature-value relationship was monitored and is shown in the Progress Monitoring panel in Figure 22.

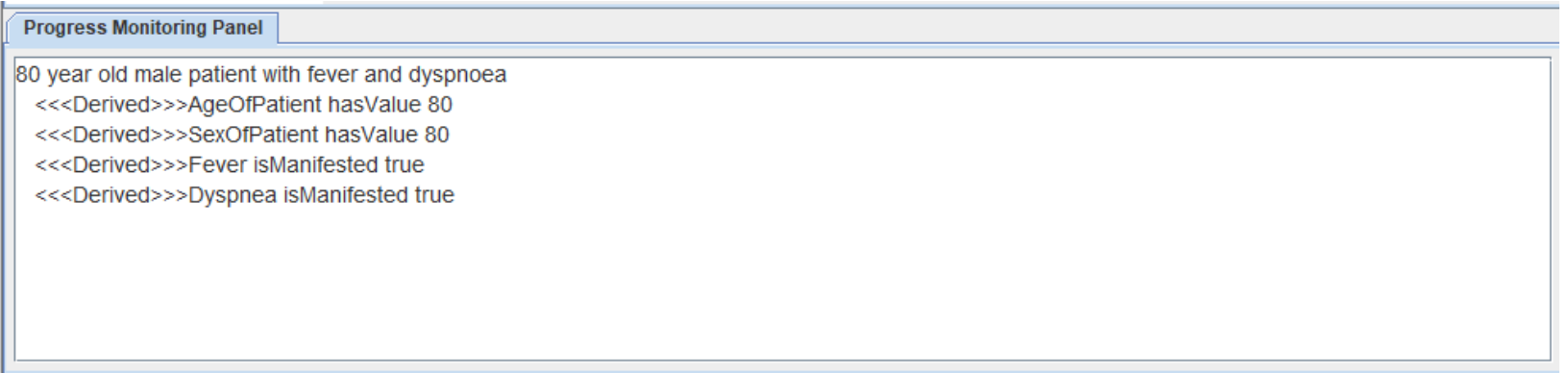

Figure 22: A demonstration of the formalization of feature-value extracted in ontology representation

The CBR-engine then collected the ontology representation of the new case for a reasoning operation which was expected to yield the output shown in Figure 23. 
Covid19: A NLP-Ontology Oriented Case-Based Framework for Early Detection and Diagnosis of Novel Coronavirus

\begin{tabular}{|c|c|c|}
\hline CBR-Engine Panel & Result/Output Panel & \\
\hline Accuracy & & 97.10144927536231 \\
\hline Sensitivity & & 0.9848484848484849 \\
\hline Specificity & & 0.6666666666666666 \\
\hline Precision & & 0.9848484848484849 \\
\hline Recall & & 0.9848484848484849 \\
\hline F1_score & & 0.9848484848484849 \\
\hline 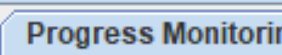 & & \\
\hline
\end{tabular}

Figure 23: Result of diagnosis of a case of COVID-19 showing the status (positive or negative), clinical diagnosis (acute, mild or severe), estimated duration (in days), and likely treatment

\subsection{Result and Discussion}

In this section, we present the performance of the proposed CBR framework compared to the performances of other similar systems. The following are the metrics and their corresponding formula used in analyzing the performance described in this section.

i. Accuracy $=(\mathrm{TP}+\mathrm{TN}) /(\mathrm{TP}+\mathrm{TN}+\mathrm{FP}+\mathrm{FN})$

ii. Specificity $=\mathrm{TN} /(\mathrm{TN}+\mathrm{FP})$

iii. Sensitivity $=\mathrm{TP} /(\mathrm{TP}+\mathrm{FN})$

iv. Precision $=\mathrm{TP} /(\mathrm{TP}+\mathrm{FP})$

v. $\mathrm{F} 1=(2 *$ Recall $) /((2 *$ Recall $)+\mathrm{FP}+\mathrm{FN})$

vi. $\mathrm{F}=(2 *$ Precision $*$ Recall $) /($ Recall + Precision $)$

vii. Recall $=\mathrm{TP} /(\mathrm{TP}+\mathrm{FN})$

Note that the following are the derivations for the TN, TP, FN, and FP:

$\mathrm{TN}=$ Suspected cases of COVID-19 which both the proposed CBR framework and the curated dataset presented concluded to be negative cases of COVID-19.

$\mathrm{TP}=$ Suspected cases of COVID-19 which both the proposed CBR framework and the curated dataset presented as being positive with COVID-19.

FN = Suspected cases of COVID-19 which the proposed CBR framework concluded to be negative cases of COVID-19 while the curated dataset presented as being positive with COVID-19. 
FP = Suspected cases of COVID-19 which the proposed CBR framework presented as being positive with COVID19, while the curated dataset shows negative cases of COVID-19.

\subsection{Comparison of the ontology of the systems with others}

The ontologies developed in the research are very tangible in enhancing the performance of the proposed CBR framework. However, to measure the performance and importance of the knowledge representation formalism used in this study, we resolved to compare the efficiency of the proposed ontology with other related ontologies used in related studies on CBR by using the following metrics:

i. Class Complexity: Average number of paths to reach a class from the Thing class

ii. Class Complexity: Average number of semantic relations for object properties per class

iii. Abstraction: Average depth of the ontology

iv. Cohesion: Average number of connected classes

v. Semantic richness: Ratio of total number of semantic relations mapped to classes, by all ontology relations consisting of object properties and subsumption relations.

vi. Inheritance richness: Average number of subclasses in a class.

vii. Attribute richness: Ratio of total number of data type properties by the number of classes.

viii. Comprehension of properties (object and data type): Percentage of annotation of the properties in the ontology

ix. Comprehension of classes: Percentage of annotation of the classes in the ontology

Based on these metrics, the performance measurements in the following subsections are presented. Figure 24 shows how the values used in computing the metrics were derived through the Protégé while viewing the ontology.

\begin{tabular}{|c|c|c|c|}
\hline Ontology metrics: & & Ontology metrics: & \\
\hline Metrics & & Reflexive $\mathrm{bjectProperty}$ & 0 \\
\hline & & Irrefexive ObjectProperty & 0 \\
\hline Axiom & 798 & ObjectPropertyDomain & 14 \\
\hline Logical axiom count & 531 & ObjectPropertyRange & 24 \\
\hline Declaration axioms count & 260 & SubPropertychainof & 0 \\
\hline Class count & 146 & & \\
\hline Object property count & 14 & Data property axioms & \\
\hline Data property count & 15 & SubDataPropertyOf & 1 \\
\hline Individual count & 85 & EquivalentDataProperties & 0 \\
\hline Annotation Property count & 2 & DisjointDataProperties & 0 \\
\hline & & FunctionalDataProperty & 0 \\
\hline Class axioms & & DataPropertyDomain & 193 \\
\hline SubClass Of & 139 & DataPropertyRange & 12 \\
\hline EquivalentClasses & 0 & Individual axioms & \\
\hline DisjointClasses & 11 & Classassertion & 69 \\
\hline $\mathrm{GCl}$ count & 0 & ghasabsention & 69 \\
\hline Hidden $\mathrm{GCl}$ Count & 0 & Ujectiroperty/Assertion & 21 \\
\hline
\end{tabular}

Figure 24: An outline of Protégé-based metrics which yielded values for computation of the comparison metrics used in Tables 3 and 4.

Table 3: An evaluation of some related ontologies used in similar CBR studies in comparison with the proposed ontology as shown in Figures 15 and 16

\begin{tabular}{|c|c|c|c|c|c|c|c|c|c|}
\hline \multirow{3}{*}{$\begin{array}{l}\text { Metrics } \\
\text { Studies } \\
\text { (Year) } \\
\text { [Ref] } \\
\end{array}$} & \multicolumn{9}{|c|}{ Ontology Metrics } \\
\hline & \multicolumn{2}{|c|}{ Complexity } & \multirow{2}{*}{$\begin{array}{l}\text { Abstractio } \\
\text { n }\end{array}$} & \multirow{2}{*}{$\begin{array}{l}\text { Cohesio } \\
\text { n }\end{array}$} & \multicolumn{3}{|c|}{ Conceptualization richness } & \multicolumn{2}{|c|}{ Comprehension } \\
\hline & $\begin{array}{c}\text { Clas } \\
\mathrm{s}\end{array}$ & $\begin{array}{c}\text { Propertie } \\
\text { s }\end{array}$ & & & $\begin{array}{c}\text { Semanti } \\
\text { c }\end{array}$ & $\begin{array}{c}\text { Data } \\
\text { propert } \\
y\end{array}$ & $\begin{array}{c}\text { Inheritanc } \\
\mathrm{e}\end{array}$ & $\begin{array}{c}\text { Classe } \\
\text { s }(\%)\end{array}$ & $\begin{array}{c}\text { Propert } \\
\mathrm{y}(\%)\end{array}$ \\
\hline $\begin{array}{l}\text { Proposed } \\
\text { framewor } \\
\text { k }\end{array}$ & 4 & 2 & 4 & 181 & 1.041 & 0.04 & 8.2 & 10 & 10 \\
\hline
\end{tabular}




\begin{tabular}{|c|c|c|c|c|c|c|c|c|c|}
\hline $\begin{array}{l}\text { El- } \\
\text { Sappagh } \\
\text { and } \\
\text { Elmogy } \\
{[63]}\end{array}$ & 5 & 1.4 & 2 & 63 & 0.495 & 2.26 & 5.0 & 88.71 & $2.04 \%$ \\
\hline $\begin{array}{l}\text { Heras, et } \\
\text { al. [64] }\end{array}$ & 3 & 1.3 & 2 & 27 & 0.62 & 0.92 & 2.875 & 0.0 & 0.0 \\
\hline
\end{tabular}

The results of Tables 3 and 4 shows the richness of the axioms, properties (object and data type) and instances of the proposed ontology used in this study.

Table 4: An evaluation of some related ontologies based on the contents of their terminology box (Tbox)

\begin{tabular}{|c|c|c|c|c|c|c|c|}
\hline \multicolumn{8}{|c|}{ Metrics } \\
\hline $\begin{array}{l}\text { Studies } \\
\text { (Year) } \\
\text { [Ref] }\end{array}$ & $\begin{array}{l}\text { Number } \\
\text { of } \\
\text { individuals }\end{array}$ & $\begin{array}{l}\text { Properties } \\
\text { with } \\
\text { domain/range } \\
\quad(\%)\end{array}$ & $\begin{array}{l}\text { Number } \\
\text { of } \\
\text { properties }\end{array}$ & \begin{tabular}{l} 
Documentation \\
of \\
properties \\
\multicolumn{1}{c}{$(\%)$} \\
\end{tabular} & $\begin{array}{l}\text { No. of } \\
\text { classes }\end{array}$ & $\begin{array}{l}\text { Documentation } \\
\text { of the classes } \\
(\%)\end{array}$ & $\begin{array}{l}\text { No. } \\
\text { Axioms }\end{array}$ \\
\hline $\begin{array}{l}\text { Proposed } \\
\text { ontology }\end{array}$ & 202 & $100 / 100$ & 19 & 10 & 196 & 10 & 1078 \\
\hline $\begin{array}{l}\text { El- } \\
\text { Sappagh } \\
\text { and } \\
\text { Elmogy } \\
{[63]}\end{array}$ & 2640 & $\begin{array}{l}98.47 \% / \\
98.98 \%\end{array}$ & 196 & 2.04 & 62 & 88.71 & 1316 \\
\hline $\begin{array}{l}\text { Heras, et } \\
\text { al. [64] }\end{array}$ & 0 & $85.48 / 77.41$ & 62 & 0 & 26 & 0 & 446 \\
\hline
\end{tabular}

\subsection{Presentation of the accuracy, sensitivity and specificity of the proposed approach}

In this section, we present the performance of the proposed CBR model using diagnosis metrics like accuracy, specificity, sensitivity, precision, recall, and F1-score. The choice of these metrics was informed by the peculiarity of the relationship of the values with the disease. For instance, diagnostic accuracy metric was used to evaluate the ability of a diagnostic test to correctly identify a target condition (COVID-19 in this case). This metric particularly is very applicable to cases of diagnoses in medicine since it allows for increased confidence and acceptability of results. In addition, accuracy of diagnosis could help to determine the difference between life and death, so that a system which outperforms another may be seen from an improved accuracy, which also leads to reliability of diagnoses results. Other metric considerations for performance measure in this study were sensitivity and specificity which are also referred to as True Positive Rate (TPR) and True Negative Rate (TNR) respectively. Sensitive and specificity of our system as shown in Table 5, implies the number of COVID-19 cases withthe condition who had a positive result, and the number of COVID-19 cases who did not have the disease and had a negative result respectively. The relationship between these two metrics with respect to accuracy of diagnosis is that the latter allows for the evaluation of the former.

Looking at the results of the metrics above as presented in Table 5, we discovered the proposed CBR framework had a good accuracy. In addition, the sensitivity and specificity value indicated that our system was able to correctly classify cases of COVID-19 as either positive or negative respectively. The precision value means that an average of 2 COVID-19 cases can be effectively detected by the proposed CBR framework as negative while the remaining 8 out of 10 cases are positive. Similarly, the recall value is $99 \%$ which means that approximately 10 out of 10 cases of COVID-19 are correctly classified as positive. F1 score presents the ability of our framework to classify the cases of the disease since the metrics represents a harmonious mean of precision and recall. The precision and recall results therefore portray relevance of positive cases and proportion of correct positive cases are respectively. These are sometimes referred to as Positive Predictive Value (PPV) and True Positive Rate (TPR) respectively. 
Table 5: Performance evaluation of the proposed CBR framework using the accuracy, sensitivity, specificity, precision, recall and F1 score

\begin{tabular}{lrrrrrr} 
& & & \multicolumn{2}{c}{ Performance Metrics } & Recall & \multicolumn{2}{l}{ F1 } \\
Accuracy & Sensitivity & Specificity & Precision & Score \\
Values & $97.10 \%$ & 0.98 & 0.66 & 0.984 & 0.984 & 0.98 \\
\hline
\end{tabular}

In the next section, we shall compare the performance recorded by the proposed CBR framework with similar studies.

\subsection{Comparing the proposed approach with similar methods}

A comparative analysis of the performance of our proposed approach was carried out with other case-based reasoning studies. Although the domain of application of the CBR models reviewed differs (medical and nonmedical), we discovered that the most important factor lay in the formalism of cases and condition and similarity measures for retrieval of similar cases. An approach of CBR with dominance in the list of studies reviewed using fuzzy logic and those whose cases used ontology for formalism purpose. Also, we observed that some studies investigated the peculiarity and importance of different similarity metrics like Euclidean distance, cosine similarity and others. The effect of such choice of similarity measure helped them to discover the performance effect of a selected metric. The decision of the selection of distance/similarity measure is sometimes influenced by the formalism in which case features are represented. Considering the wide adoption of the use of ontologies as a tool for formalizing cases and its features, we discovered that the interesting performance of this study must have drawn much benefit from the ontology approach for knowledge modeling. An interesting consideration made in this study which makes it outperform other similar works is the choice of a semantic and ontology-based similarity measure metric. We observed that this allowed for a better comparison of cases during retrieval.

Furthermore, the novelty of the approach proposed in this study was also uncovered as we compared it with similar studies in the last decade. Only our study adopted the use of NLP technique in extraction of features represented in a presenting case. This allowed for a non-partial automation of the process of diagnosis/detection/classification of cases. We argue that such approach allows for an increase in the level of acceptance of the CBR paradigm. This deduction was made based on the popular manual approach for the extraction of cases and their features from documents represented using natural language. Although some fuzzy-CBR frameworks which were reviewed and compared in Table 6 demonstrate good performance, they are, however, surpassed by our model which combines the techniques of NLP and machine learning (sentiment analysis) in extraction of features in any presenting case. As a result, we presume that an investigation into the hybridization of a CBR model using fuzzy logic, NLP and ontologies may yield a very encouraging performance, and thereby position CBR paradigm as a competitive option for reasoning tasks in artificial intelligence (AI).

In any medical system, the result of diagnosis is more important because the patient has so much to lose when there is a misdiagnosis. So, both under-diagnosis and over-diagnosis are both errors in medical systems and have been a source of concern to wide acceptance for AI-based diagnostic and detection systems in medicine. While overdiagnoses may have to do with over stating the condition of diagnosed case, under-diagnosis is a condition where a diagnosed case does not go on to cause any symptoms or ill-health. This can result in the blurring of the borders between health and disease. Therefore, a diagnostic accuracy helps to investigate how well a particular diagnostic test is able to identify a target condition, in comparison to a reference test. In this study, we carried out our comparison of the proposed CBR framework with other similar studies using diagnostic accuracy. As seen in Table 6 , the accuracy of our framework outperforms those of previous studies we compared. Most interesting is the capability of our CBR framework to detect the novel coronavirus (COVID-19) at a higher accuracy. This, therefore, positions this study as a candidate for further improvement of CBR models in future works seeking to diagnose any family of the Covs diseases.

Table 6: A summary of some case-based reasoning (CBR) models and framework, and their domains of application, approaches/techniques used, description of approach and accuracy of the systems 


\begin{tabular}{|c|c|c|c|c|}
\hline Studies [Ref] & Year & $\begin{array}{l}\text { Approach used for } \\
\text { reasoning or diagnoses }\end{array}$ & Domain of Application & $\begin{array}{c}\text { Accuracy } \\
(\%)\end{array}$ \\
\hline $\begin{array}{l}\text { Proposed } \\
\text { framework }\end{array}$ & 2020 & $\begin{array}{l}\text { CBR and NLP, and } \\
\text { Semantic Web }\end{array}$ & $\begin{array}{l}\text { Detection and diagnosis of COVID-19 } \\
\text { (Novel Coronavirus) }\end{array}$ & 97.10 \\
\hline $\begin{array}{l}\text { Rahim et al. } \\
{[65]}\end{array}$ & 2019 & Traditional CBR & Diagnosis of psychological disorders & - \\
\hline $\begin{array}{l}\text { Zhong et al. } \\
\text { [66] }\end{array}$ & 2018 & Text-CBR and ontology & $\begin{array}{l}\text { Non-medical: Fault diagnosis and } \\
\text { predication by cloud computing }\end{array}$ & - \\
\hline $\begin{array}{l}\text { Zhang et al. } \\
\text { [67] }\end{array}$ & 2017 & Traditional CBR & $\begin{array}{l}\text { Non-medical: Theory of inventive } \\
\text { problem solving for inventive design }\end{array}$ & - \\
\hline $\begin{array}{l}\text { El-Sappagh } \\
\text { and Elmogy } \\
\text { [63] }\end{array}$ & 2015 & $\begin{array}{l}\text { Fuzzy-CBR, } \\
\text { Ontologies }\end{array}$ & Diabetics & 97.67 \\
\hline $\begin{array}{l}\text { Shen et al. } \\
{[68]}\end{array}$ & 2015 & $\begin{array}{l}\text { CBR with ontology } \\
\text { approach }\end{array}$ & Diagnosis of gastric cancer & - \\
\hline $\begin{array}{l}\text { Heras et al. } \\
{[64]}\end{array}$ & 2013 & $\begin{array}{l}\text { CBR with ontology } \\
\text { approach }\end{array}$ & Non-medical: multi-agent systems & - \\
\hline $\begin{array}{l}\mathrm{Li} \text { and } \mathrm{Ho} \\
{[69]}\end{array}$ & 2009 & CBR and fuzzy logic & $\begin{array}{l}\text { Non-medical: Prediction of financial } \\
\text { activity }\end{array}$ & 92.36 \\
\hline $\begin{array}{l}\text { Petrovic et. } \\
\text { al. }[70]\end{array}$ & 2011 & Traditional CBR & Radiotherapy planning & 84.72 \\
\hline $\begin{array}{l}\text { Fan et. al. } \\
{[71]}\end{array}$ & 2009 & CBR, Fuzzy decision tree & $\begin{array}{l}\text { Medical data classification: breast } \\
\text { cancer and liver disorders }\end{array}$ & $\begin{array}{c}98.40 \text { and } \\
81.60\end{array}$ \\
\hline $\begin{array}{l}\text { Begum et. al. } \\
{[72]}\end{array}$ & 2009 & CBR and fuzzy logic & Medical data for diagnosis of stress & 90.00 \\
\hline
\end{tabular}

\subsection{Conclusion}

This study is largely focused on adapting a CBR concept to the problem of classifying cases of COVID-19 as either positive or negative even when the disease is in its early stage in the presented case. An NLP model for feature extraction of a presented case was designed and implemented. The innovation of the work presented here lies in sentence-level extraction of feature-value pair for all a-priori declared features. Furthermore, the case retrieval similarity metric applied to the CBR framework proposed in this study contributed to the interesting performance of the system. Meanwhile, knowledge representation (archive of cases stored in the CBR) in the proposed framework was achieved using ontology-based knowledge formalization technique. In addition, new cases were also formalized using ontologies so as to allow for a homogenous basis for case comparisons. The result obtained shows that our proposed framework outperformed state-of-the-art CBR studies with similar approaches to the one in this study. In future, we intend to investigate the performance of our retrieval algorithm over different similarity/distance measure metrics. This will allow for future studies using ontologies and CBR paradigm to effectively select or even combine similarity metrics. Also, we intend to hybridize the proposed method with machine learning methods which allow for application classification algorithm such as SVM.

\section{References}

1. Naudé, Wim, Artificial Intelligence Against Covid-19: An Early Review. IZA Discussion Paper No. 13110. Available at SSRN: https://ssrn.com/abstract=3568314

2. Pirouz, B., Shaffiee Haghshenas, S., Shaffiee Haghshenas, S. and Piro, P., 2020. Investigating a Serious Challenge in the Sustainable Development Process: Analysis of Confirmed cases of COVID-19 (New Type of Coronavirus) Through a Binary Classification Using Artificial Intelligence and Regression Analysis. Sustainability, 12(6), p.2427.

3. Al-qaness, M.A., Ewees, A.A., Fan, H. and Abd El Aziz, M., 2020. Optimization method for forecasting confirmed cases of COVID-19 in China. Journal of Clinical Medicine, 9(3), p.674.

4. Ting, D.S.W., Carin, L., Dzau, V. and Wong, T.Y., 2020. Digital technology and COVID-19. Nature Medicine, pp.1-3. 
5. Vaishya, R., Javaid, M., Khan, I.H. and Haleem, A., 2020. Artificial Intelligence (AI) applications for COVID19 pandemic. Diabetes \& Metabolic Syndrome: Clinical Research \& Reviews.

6. Leung, G.M. and Leung, K., 2020. Crowdsourcing data to mitigate epidemics. The Lancet Digital Health.

7. oton, P. A. 1988. Using Experience in Learning and Problem Solving. MIT Press.

8. Bareiss, E. R. 1989.Exemplar-Based Knowledge Acquisition: A unified Approach to Concept Representation, Classification and Learning. 300 North Zeeb road, Ann Arbor, MI 48106-1346: UMI.

9. Gierl, L., Bull, M. and Schmidt, R., 1998. CBR in Medicine. In Case-Based Reasoning Technology (pp. 273297). Springer, Berlin, Heidelberg.

10. Blanco, X., Rodríguez, S., Corchado, J.M. and Zato, C., 2013. Case-based reasoning applied to medical diagnosis and treatment. In distributed computing and artificial intelligence (pp. 137-146). Springer, Cham.

11. Vásquez-Morales, G.R., Martínez-Monterrubio, S.M., Moreno-Ger, P. and Recio-García, J.A., 2019. Explainable Prediction of Chronic Renal Disease in the Colombian Population Using Neural Networks and Case-Based Reasoning. IEEE Access, 7, pp.152900-152910.

12. Benamina, M., Atmani, B. and Benbelkacem, S., 2018. Diabetes diagnosis by case-based reasoning and fuzzy logic. IJIMAI, 5(3), pp.72-80.

13. COVID-19 WHO global report: https://covid19.who.int/ [Accessed 21 April, 2020]

14. Li, Q., Guan, X., Wu, P., Wang, X., Zhou, L., Tong, Y., Ren, R., Leung, K.S., Lau, E.H., Wong, J.Y. and Xing, X., 2020. Early transmission dynamics in Wuhan, China, of novel coronavirus-infected pneumonia. New England Journal of Medicine.

15. Wu, J.T., Leung, K. and Leung, G.M., 2020. Nowcasting and forecasting the potential domestic and international spread of the 2019-nCoV outbreak originating in Wuhan, China: a modelling study. The Lancet, 395(10225), pp.689-697.

16. Johansson, M.A., Apfeldorf, K.M., Dobson, S., Devita, J., Buczak, A.L., Baugher, B., Moniz, L.J., Bagley, T., Babin, S.M., Guven, E. and Yamana, T.K., 2019. An open challenge to advance probabilistic forecasting for dengue epidemics. Proceedings of the National Academy of Sciences, 116(48), pp.24268-24274.

17. Tuite, A.R. and Fisman, D.N., 2020. Reporting, epidemic growth, and reproduction numbers for the 2019 novel coronavirus (2019-nCoV) epidemic. Annals of Internal Medicine.

18. Mizumoto, K., Kagaya, K., Zarebski, A. and Chowell, G., 2020. Estimating the asymptomatic proportion of coronavirus disease 2019 (COVID-19) cases on board the Diamond Princess cruise ship, Yokohama, Japan, 2020. Eurosurveillance, 25(10), p.2000180.

19. Liu, Y., Gayle, A.A., Wilder-Smith, A. and Rocklöv, J., 2020. The reproductive number of COVID-19 is higher compared to SARS coronavirus. Journal of travel medicine.

20. Hu, Z., Ge, Q., Jin, L. and Xiong, M., 2020. Artificial intelligence forecasting of covid-19 in China. arXiv preprint arXiv:2002.07112.

21. Funk, S., Camacho, A., Kucharski, A.J., Eggo, R.M. and Edmunds, W.J., 2018. Real-time forecasting of infectious disease dynamics with a stochastic semi-mechanistic model. Epidemics, 22, pp.56-61.

22. Johansson, M.A., Apfeldorf, K.M., Dobson, S., Devita, J., Buczak, A.L., Baugher, B., Moniz, L.J., Bagley, T., Babin, S.M., Guven, E. and Yamana, T.K., 2019. An open challenge to advance probabilistic forecasting for dengue epidemics. Proceedings of the National Academy of Sciences, 116(48), pp.24268-24274.

23. Rao, A.S.R.S., Krantz, S.G., Kurien, T., Bhat, R. and Sudhakar, K., 2020. Model-Based Retrospective Estimates for COVID-19 or Coronavirus in India: Continued Efforts Required to Contain the Virus Spread. Current Science, 118(7), pp.1023-1025.

24. Buonomo, B., 2020. Effects of information-dependent vaccination behavior on coronavirus outbreak: insights from a SIRI model. Ricerche di Matematica, p.1.

25. Kim, S., Kim, Y.J., Peck, K.R. and Jung, E., 2020. School Opening Delay Effect on Transmission Dynamics of Coronavirus Disease 2019 in Korea: Based on Mathematical Modeling and Simulation Study. Journal of Korean Medical Science, 35(13). 
26. Hellewell, J., Abbott, S., Gimma, A., Bosse, N.I., Jarvis, C.I., Russell, T.W., Munday, J.D., Kucharski, A.J., Edmunds, W.J., Sun, F. and Flasche, S., 2020. Feasibility of controlling COVID-19 outbreaks by isolation of cases and contacts. The Lancet Global Health.

27. Jia, J., Ding, J., Liu, S., Liao, G., Li, J., Duan, B., Wang, G. and Zhang, R., 2020. Modeling the control of COVID-19: Impact of policy interventions and meteorological factors. ELECTRONIC JOURNAL OF DIFFERENTIAL EQUATIONS Article Number: 23 Published: MAR 162020.

28. Choi, S. and Ki, M., 2020. Estimating the reproductive number and the outbreak size of Novel Coronavirus disease (COVID-19) using mathematical model in Republic of Korea. Epidemiology and Health, p.e2020011.

29. Huang, R., Liu, M. and Ding, Y., 2020. Spatial-temporal distribution of COVID-19 in China and its prediction: A data-driven modeling analysis. The Journal of Infection in Developing Countries, 14(03), pp.246-253.

30. Gostic, K., Gomez, A.C., Mummah, R.O., Kucharski, A.J. and Lloyd-Smith, J.O., 2020. Estimated effectiveness of symptom and risk screening to prevent the spread of COVID-19. Elife, 9, p.e55570.

31. Wang, C., Cheng, Z., Yue, X.G. and McAleer, M., 2020. Risk management of COVID-19 by universities in China. JOURNAL OF RISK AND FINANCIAL MANAGEMENT Volume: 13 Issue: 2 Article Number: 36 Published: FEB 2020.

32. Roosa, K., Lee, Y., Luo, R., Kirpich, A., Rothenberg, R., Hyman, J.M., Yan, P. and Chowell, G., 2020. Shortterm forecasts of the COVID-19 epidemic in Guangdong and Zhejiang, China: February 13-23, 2020. Journal of Clinical Medicine, 9(2), p.596.

33. Yang, S., Cao, P., Du, P., Wu, Z., Zhuang, Z., Yang, L., Yu, X., Zhou, Q., Feng, X., Wang, X. and Li, W., 2020. Early estimation of the case fatality rate of COVID-19 in mainland China: a data-driven analysis. Annals of Translational Medicine, 8(4).

34. Jiang, X., Coffee, M., Bari, A., Wang, J., Jiang, X., Huang, J., Shi, J., Dai, J., Cai, J., Zhang, T. and Wu, Z., 2020. Towards an artificial intelligence framework for data-driven prediction of coronavirus clinical severity. CMCComputers, Materials \& Continua, 63(1), pp.537-51.

35. Yin, F., Lv, J., Zhang, X., Xia, X. and Wu, J., 2020. COVID-19 information propagation dynamics in the Chinese Sina-microblog. Mathematical biosciences and engineering: MBE, 17(3), p.2676.

36. Zhou, W., Wang, A., Xia, F., Xiao, Y. and Tang, S., 2020. Effects of media reporting on mitigating spread of COVID-19 in the early phase of the outbreak. MATHEMATICAL BIOSCIENCES AND ENGINEERING Volume: 17 Issue: 3 Pages: 2693-2707 Published: 2020

37. Yang, C. and Wang, J., 2020. A mathematical model for the novel coronavirus epidemic in Wuhan, China. Mathematical Biosciences and Engineering, 17(3), pp.2708-2724.

38. Rong, X., Yang, L., Chu, H. and Fan, M., 2020. Effect of delay in diagnosis on transmission of COVID-19. Mathematical Biosciences and Engineering, 17(3), pp.2725-2740.

39. Hou, C., Chen, J., Zhou, Y., Hua, L., Yuan, J., He, S., Guo, Y., Zhang, S., Jia, Q., Zhao, C. and Zhang, J., 2020. The effectiveness of the quarantine of Wuhan city against the Corona Virus Disease 2019 (COVID-19): wellmixed SEIR model analysis. Journal of Medical Virology.

40. Prem, K., Liu, Y., Russell, T.W., Kucharski, A.J., Eggo, R.M., Davies, N., Flasche, S., Clifford, S., Pearson, C.A., Munday, J.D. and Abbott, S., 2020. The effect of control strategies to reduce social mixing on outcomes of the COVID-19 epidemic in Wuhan, China: a modelling study. The Lancet Public Health.

41. Ho, D., Addressing COVID-19 Drug Development with Artificial Intelligence. Advanced Intelligent Systems.

42. Shi, F., Wang, J., Shi, J., Wu, Z., Wang, Q., Tang, Z., He, K., Shi, Y. and Shen, D., 2020. Review of artificial intelligence techniques in imaging data acquisition, segmentation and diagnosis for covid-19. In IEEE Reviews in Biomedical Engineering.

43. Kim, D., Hong, S., Choi, S. and Yoon, T., 2016. Analysis of transmission route of MERS coronavirus using decision tree and Apriori algorithm. In 2016 18th International Conference on Advanced Communication Technology (ICACT) (pp. 559-565). IEEE.

44. Chahrour, M., Assi, S., Bejjani, M., Nasrallah, A.A., Salhab, H., Fares, M. and Khachfe, H.H., 2020. A bibliometric analysis of Covid-19 research activity: A call for increased output. Cureus, 12(3). 
45. Hossain, M.M., 2020. Current status of global research on novel coronavirus disease (Covid-19): A bibliometric analysis and knowledge mapping. Available at SSRN 3547824.

46. Lou, J., Tian, S.J., Niu, S.M., Kang, X.Q., Lian, H.X., Zhang, L.X. and Zhang, J.J., 2020. Coronavirus disease 2019: a bibliometric analysis and review. European Review for Medical and Pharmacological Sciences, 24(6), pp.3411-3421.

47. Dasgupta, S., Padia, A., Maheshwari, G., Trivedi, P., \& Lehmann, J. (2018). Formal Ontology Learning from English IS-A Sentences. arXiv:1802.03701v1 [cs.AI].

48. Christopher, M. D., Surdeanu, M., Bauer, J., Finkel, J., Bethard, S. J., \& McClosky, D. (2014). The Stanford CoreNLP Natural Language Processing Toolkit. In Proceedings of the 52nd Annual Meeting of the Association for Computational Linguistics: System Demonstrations, 55-60.

49. Michelen, M., Jones, N. and Stavropoulou, C., 2020. In patients of COVID-19, what are the symptoms and clinical features of mild and moderate cases? Centre for Evidence-Based Medicine https://www. cebm. net/covid-19/in-patients-of-covid-19-what-are-the-symptoms-and-clinical-features-of-mild-andmoderatecase/accessed, 16.

50. Cascella, M., Rajnik, M., Cuomo, A., Dulebohn, S. C., \& Napoli, R. D. (2020). Features, Evaluation and Treatment Coronavirus (COVID-19). StatPearls Publishing LLC.

51. Polamuri, S., 2015. Five most popular similarity measures implementation in python. Internet: http://dataaspirant. com/2015/04/11/five-most-popular-similarity-measuresimplementation-in-python.

52. Gan, M., Dou, X., \& Jiang, R. (2013). From Ontology to Semantic Similarity: Calculation of Ontology-Based Semantic Similarity. Computational Systems Biology, 2013, 11.

53. Gu, D., Liang, C., \& Zhao, H. (2017). A case-based reasoning system based on weighted heterogeneous value distance metric for breast cancer diagnosis. Artificial Intelligence in Medicine, 1-51.

54. Patel-Schneider, P.F. and Franconi, E., 2012, November. Ontology constraints in incomplete and complete data. In International Semantic Web Conference (pp. 444-459). Springer, Berlin, Heidelberg.

55. Herman, I., Horrocks, I., \& Patel-Schneider, P. F. (2012, December 11). OWL 2 Web Ontology Language Document Overview (Second Edition). Retrieved April 19, 2020, from W3C: https://www.w3.org/TR/owl2overview

56. O'Connor, M.J. and Das, A.K., 2009, October. SQWRL: a query language for OWL. In OWLED (Vol. 529, No. 2009).

57. O'Connor, M.J. and Das, A., 2009. SQWRL: a query language for OWL, OWL: experiences and directions (OWLED). In Fifth International Workshop (pp. 1-8).

58. Díaz-Agudo, B., González-Calero, P.A., Recio-García, J.A. and Sánchez-Ruiz-Granados, A.A., 2007. Building CBR systems with jCOLIBRI. Science of Computer Programming, 69(1-3), pp.68-75.

59. Kolodner, J.L., 1992. An introduction to case-based reasoning. Artificial intelligence review, 6(1), pp.3-34.

60. Althoff, K. (2012). Case-Based Reasoning and Expert Systems. Intelligent Information Systems Lab Institute of Computer Science University of Hildesheim.

61. Aamodt, A., \& Plaza, E. (1994). Case-Based Reasoning: Foundational Issues, Methodological Variations, and System Approaches. AI Communications, 7(1), 39-59.

62. Ben-Bassat, M., Beniaminy, I., \& Joseph, D. (1998). Combining Model-Based and Case-Based Expert Systems. Research Perspectives and Case Studies in System Test and Diagnosis Frontiers in Electronic Testing, 13(1), 179-205.

63. El-Sappagh, S., Elmogy, M., \& Riad, A. M. (2015). A Fuzzy-Ontology Oriented Case Based Reasoning Framework for Semantic Diabetics Diagnosis. Artificial Intelligence in Medicine, 1-30.

64. Heras, S., Botti, V., \& Juliana, V. (2013). A knowledge representation formalism for Case-Based-Reasoning. Agreement Technol, 105-119.

65. Rahim, R., Purba, W., Khairani, M. and Rosmawati, R., 2019, November. Online Expert System for Diagnosis Psychological Disorders Using Case-Based Reasoning Method. In Journal of Physics: Conference Series (Vol. 1381, No. 1, p. 012044). IOP Publishing. 
66. Zhong, Z., Xu, T., Wang, F. and Tang, T., 2018. Text Case-Based Reasoning Framework for Fault Diagnosis and Predication by Cloud Computing. Mathematical Problems in Engineering, 2018.

67.Zhang, P., Essaid, A., Zanni-Merk, C. and Cavallucci, D., 2017. Case-based reasoning for knowledge capitalization in inventive design using latent semantic analysis. Procedia computer science, 112, pp.323-332.

68. Shen, Y., Colloc, J., Jacquet-Andrieu, A. and Lei, K., 2015. Emerging medical informatics with case-based reasoning for aiding clinical decision in multi-agent system. Journal of biomedical informatics, 56, pp.307-317.

69. Li, S.T. and Ho, H.F., 2009. Predicting financial activity with evolutionary fuzzy case-based reasoning. Expert Systems with Applications, 36(1), pp.411-422.

70. Petrovic, S., Mishra, N. and Sundar, S., 2011. A novel case based reasoning approach to radiotherapy planning. Expert Systems with Applications, 38(9), pp.10759-10769.

71. Fan, C.Y., Chang, P.C., Lin, J.J. and Hsieh, J.C., 2011. A hybrid model combining case-based reasoning and fuzzy decision tree for medical data classification. Applied Soft Computing, 11(1), pp.632-644.

72. Begum, S., Ahmed, M.U., Funk, P., Xiong, N. and Von Schéele, B., 2009. A case-based decision support system for individual stress diagnosis using fuzzy similarity matching. Computational Intelligence, 25(3), pp.180-195. 